\title{
EL USO DE LA VARISCITA EN HISPANIA DURANTE LA ÉPOCA ROMANA. ANÁLISIS DE COMPOSICIÓN DE OBJETOS DE ADORNO Y TESELAS DE LA ZONA NOROCCIDENTAL DE LA MESETA NORTE
}

\author{
THE USE OF VARISCITA IN ROMAN HISPANIA. COMPOSITIONAL ANALYSIS OF \\ ORNAMENTS AND TESSERAE FROM THE WEST PART OF THE NORTHERN MESETA
}

\author{
JAIME GUTIÉRREZ PÉREZ* \\ RODRIGO VILLALOBOS GARCÍA** \\ CARLOS P. ODRIOZOLA***
}

\begin{abstract}
Resumen: Presentamos en este trabajo los resultados obtenidos al analizar un número significativo de cuentas de collar y teselas verdes de época romana en Palencia, León y Zamora, que, en su mayoría, resultan ser variscita. Durante época romana se diferencian dos momentos en la manufactura de esta piedra semipreciosa. En el Alto Imperio se utilizó para la realización de adornos personales, mientras que en el Bajo Imperio ese uso quedó restringido, casi en exclusividad, a la fabricación de teselas. Se comparan, además, los datos de composición de los materiales arqueológicos analizados con los resultados de las minas antiguas de variscita peninsulares, con objeto de discriminar su área de procedencia.
\end{abstract}

Palabras claves: Análisis de composición, época romana, glyptica, Meseta Norte, teselas, variscita, villas tardías.

La variscita, un fosfato de aluminio hidratado, es un mineral "raro" que ha sido empleado profusamente durante ciertos periodos de la historia de Europa Occidental. Las cualidades físicas de esta piedra

\footnotetext{
* Departamento de Prehistoria, Arqueología, Antropología Social y CC. y TT. Historiográficas, Universidad de Valladolid. Correo-e: jaguper82@hotmail.com (responsable de la correspondencia). Teléfono: 654035987
}

\begin{abstract}
This paper focuses on scientific analysis of Roman green beads and tesserae from Palencia, León and Zamora. Almost all the analysed materials resulted to be performed in variscite mineral. During Roman times there are two different moments of use of this semiprecious stone: Principate, when it was used to perform body ornamentation, and Late Roman Empire, when its use was limited to tesserae production. We have also performed source analysis by comparing the obtained compositional data with Iberian ancient mines data.

Keywords: X-ray fluorescence, Roman times, glyptica, Northern Plateau, tesserae, variscite, Late Roman villas.
\end{abstract}

semipreciosa (dureza de 4-5, color verde vivo, brillo céreo) la hacen muy apropiada para su uso en la elaboración de adornos lo cual, según nos ilustra el registro

\footnotetext{
** Departamento de Prehistoria, Arqueología, Antropología Social y CC. y TT. Historiográficas, Universidad de Valladolid. Correo-e: rodrigovillalobosgarcia@gmail.com

*** Departamento de Prehistoria y Arqueología, Universidad de Sevilla. Correo-e: codriozola@us.es.
} 
arqueológico, ha sucedido a lo largo de los primeros compases de la Prehistoria Reciente (Edo i Benaiges et al. 1995; Domínguez-Bella 2004; Herbaut y Querré 2004; Odriozola et al. 2010; Villalobos 2012) y también en otros momentos como la Edad Moderna (García-Guinea et al. 2000) o la Época Romana. En esta última se conoce su utilización tanto en el Alto como en el Bajo Imperio así como en la mitad Occidental y en la Oriental del Imperio, fundamentalmente para la elaboración de elementos de adorno como cuentas prismáticas hexagonales (Sanz et al. 1990; Hutchinson 1996; Middleton et al. 2007; Frantz et al. 2009) $\mathrm{y}$, también, para su transformación en teselas para la realización de los pavimentos musivarios que decoran, a modo de alfombra, los suelos de las villas tardías (Marcos Fierro 1994).

\section{LA MINERÍA DE VARISCITA EN LA HISPANIA ROMANA}

En la península ibérica se conocen tres grandes afloramientos de variscita con evidencias de minería antigua: Las Cercas en Palazuelo de las Cuevas, Zamora (Arribas et al. 1971); Can Tintorer en Gavà, Barcelona (Villalba et al. 1986) y Pico Centeno en Encinasola, Huelva (Odriozola et al. 2010); así como otros de entidad más modesta o sin evidencias de explotación minera en Tras-os-Montes, Portugal (Meireles et al. 1987) o Sansenxo, Pontevedra (Moro et al. 1995). Esta escasez es semejante a la constatada para el resto de Europa Occidental, pues sólo se conocen afloramientos en Saint-Austell, Reino Unido; Pannacé y Montrebras, Francia y, por último, Sarrabus, en Cerdeña (Marini et al. 1989; Massé 1971; Forestier et al. 1973a; Forestier et al. 1973b, Lheur 1993; Balagny 1939; Barstow 1982; Elton 1996).

Las Cercas, en Palazuelo de las Cuevas (Zamora), fue el primer afloramiento de variscita descubierto en la península ibérica, momento en el que se identificaron una serie de evidencias de minas antiguas que, en un primer momento, fueron adscritas a época islámica en base a argumentos filológicos (Arribas et al. 1971). Sin embargo, trabajos arqueológicos posteriores no hallaron ninguna prueba material de su explotación medieval descubriéndose, en cambio, un taller de lapidario anejo a las minas que presentaba cerámicas romanas altoimperiales junto con restos de producción de adornos de variscita (Sanz et al. 1990).

Las minas de Can Tintorer, en Gavà (Barcelona) son un célebre yacimiento descubierto en la década de los 70 del s. XX (Alonso et al. 1978) el cual, gracias a la excavación arqueológica de casi un centenar de estructuras subterráneas, ha ofrecido abundantes y claras evidencias de su cronología neolítica (Villalba et al. 1986; Bosch y Borrell 2009). Otras evidencias mineras en este mismo espacio apuntan a una cronología iberorromana, entre los siglos III a.C. y I d.C., pero en este caso el interés parece haberse orientado exclusivamente hacia los minerales de hierro (Bordas et al. 2009). Aunque si bien no existen pruebas sobre la explotación iberorromana de la variscita, las actividades extractivas de esta época desarrolladas en el lugar nos permiten deducir que dichas mineralizaciones serían sin duda conocidas entonces.

Por último, resta hablar de Pico Centeno, en Encinasola (Huelva). Entre los afloramientos de mineral verde conocidos en este lugar (Moro et al. 1995) han podido identificarse varias evidencias de minería antiguas entre las que se presentaban martillos de piedra prehistóricos (Nocete y Linares 1999; Odriozola et al. 2010). En estos abruptos parajes existen varias evidencias de actividades mineras romanas, así como algún lugar de hábitat (Pérez 2010), por lo que no hay que descartar que fuera también conocido.

\section{EL USO DE LA VARISCITA EN ÉPOCA ROMANA}

\subsection{La Glíptica}

El trabajo de la variscita durante la época romana, para la elaboración de adornos personales, habría que incluirla dentro de la glíptica (entallar, esculpir; en latín scalpere), es decir, el arte de trabajar los minerales, las piedras duras, las semipreciosas, etc. Con el tiempo la utilización de la variscita como elemento de adorno pasará a ser sustituida por el uso de la pasta vítrea, como se podrá observar a lo largo de este artículo.

Hemos podido analizar un número representativo de adornos y restos de producción de variscita procedentes de seis yacimientos de época altoimperial (aunque alguno de ellos tienen pervivencia, residual, en épocas posteriores), cuatro de Zamora (Palazuelos de las Cuevas, Viñas de Aliste, Moreruela de Tábara y Petauonium) y dos de León (Asturica Augusta y Lancia) (fig. 1). Además, hemos realizado una serie de lecturas sobre cuatro mosaicos de villas bajoimperiales. Dos de ellos son palentinos: "Aquiles en Skyros" (La Olmeda, Pedrosa de la Vega), único de los cuatro que se conserva in situ, y "Océano y las Nereidas" (procedente 


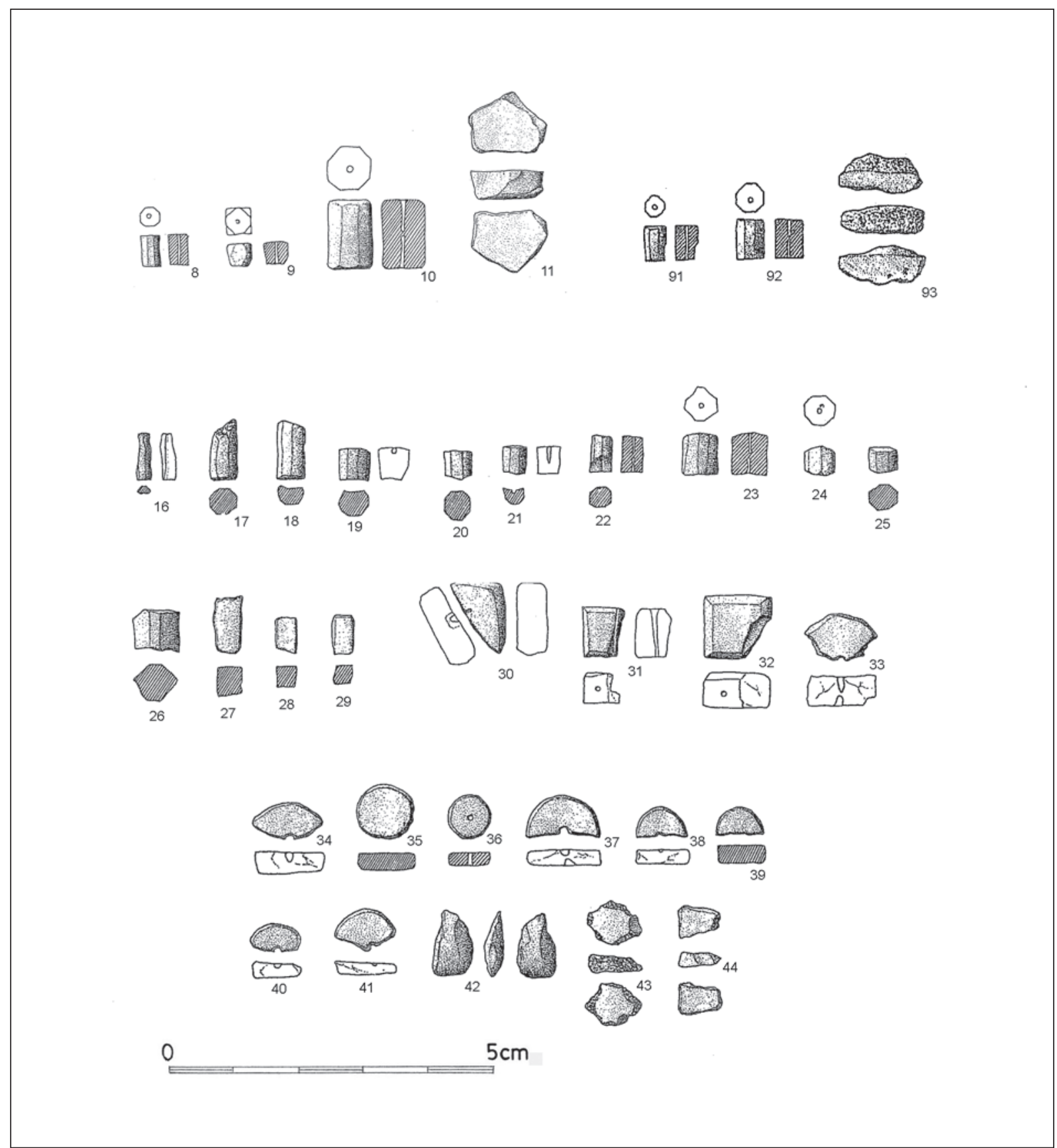

Figura 1. Cuentas y otros productos de variscita de diversos yacimientos de la provincia de Zamora estudiados en el artículo. Dehesa de Misleo (números 8 a 11), El Castrico (números 91 a 93) y El Castro (números 16 a 44), todas ellas según Sanz et alii 1990: figs. 2, 3 y 6. Se ha mantenido la numeración de la publicación original.

de Villa Possidica, Dueñas), visitable, actualmente, en el Museo Arqueológico de Palencia. Los otros dos son leoneses y están expuestos en el Museo Arqueológico de León: "Hilas y las Ninfas" (Quintana del Marco) y "Océanos" (Milla del Río).

\subsubsection{El Castro (Palazuelo de las Cuevas, Zamora)}

El yacimiento se localiza a un kilómetro al este/sureste del núcleo poblacional, sobre una pequeña loma al borde del río Aliste. En el terreno se recogieron 
abundantes fragmentos de TSH, destacando la Drag. 37 entre las formas decoradas, y la Drag. 15/17 y Ritt. 8 entre las formas lisas. Además se recuperó un gran número de elementos de variscita, ya fuesen trabajados o sin trabajar (Sanz et al. 1990: 752-755). Por la cerámica recuperada en el yacimiento, el mismo se puede fechar entre el siglo I y el siglo III de nuestra era.

Cerca de este yacimiento se delimitan las minas de variscita, a las cuales ya nos hemos referido con anterioridad, que presentan una explotación desde antiguo y de una manera, más o menos, continua. Dichas minas abastecerían al poblado en donde, con toda seguridad, se realizarían trabajos de manufactura de dicho producto. Por este motivo aparecen cuentas acabadas y/o en proceso, y/o "errores" de fábrica, además de variscita en "bruto" (Sanz et al. 1990: 761-764).

\subsubsection{El Castrico (Viñas de Aliste, Zamora)}

El yacimiento conocido como El Castrico se sitúa frente al castro de La Almena, en la margen izquierda del arroyo Campanza, sobre una amplia loma en el nivel superior del altiplano. En superficie se recogieron abundantes fragmentos de cerámica elaborada a torno de manera tosca, vidrio, sigillata y variscita. Dejando a un lado los escasos materiales que podrían sugerir una ocupación prerromana, la mayoría de las piezas recogidas indican una clara tipología romana que va desde el s. I d.C. al V d.C., predominando los asignables a los ss. I-II d.C. (Sanz et al. 1990: 755-761).

\subsubsection{Dehesa de Misleo (Moreruela de Tábara, Zamora)}

Localizado en la margen derecha del río Esla, y anegado en la actualidad una parte por un ramal del pantano, las evidencias arqueológicas se centran en el área más elevada. Los materiales que presenta este yacimiento son Terra Sigillata Hispánica, Terra Sigillata Hispánica Tardía (en número reducido), monedas de época romana, variscita (destaca la presencia del mineral tanto en estado puro como semielaborado o en piezas conclusas), etc. (Sanz et al. 1990: 750-752). El estudio de este material fue abordado por Martín Valls y Delibes (1979: 128-135), quienes apuntan a una cronología centrada en los ss. II y III d.C.

\subsubsection{Petauonium (Rosinos de Vidriales, Zamora)}

Campamento legionario de época romana, al lado del cual se instaló una auténtica ciudad para prestar diferentes servicios a los legionarios. Tiene un amplio margen cronológico que se extiende desde el siglo I a.C. hasta el siglo IV d.C., cuando las antiguas dependencias legionarias son reutilizadas de una manera residual (Carretero 1991; Carretero 2009). Durante sus excavaciones se recuperaron una serie de cuentas de collar que se asignaron a variscita.

\subsubsection{Asturica Augusta (Astorga, León).}

Ciudad de época prerromana, hipótesis por el momento sin fundamento arqueológico que se apoya en las menciones de autores clásicos como Plinio o Ptolomeo, quienes la consideran la capital de las 22 tribus de astures y sede del asentamiento de los Amacos (Plinio 3,28; Ptolomeo II, 6, 35), y cuya importancia está relacionada con la presencia de un destacamento de la Legio $X$ Gemina a raíz de las guerras cántabras. Una vez finalizada la contienda el núcleo astur fue romanizado, obteniendo un gran desarrollo que viene acompañado de dos fenómenos. Por un lado, el apogeo de las explotaciones mineras de Las Médulas y de la zona del piedemonte del Teleno (en el entorno más próximo a Astorga se localizan varios castros y explotaciones mineras, como las de Pedredo y Rabanal del Camino), cuya riqueza será administrada desde Astorga. Y por otro, la presencia de tropas romanas, quienes construyen las infraestructuras necesarias para la extracción y el transporte de los minerales (Burón 1997: 15-21). Presenta numerosos vestigios de época altoimperial (cloacas, calles, basílica, foro, termas, muralla, etc.) y parece sufrir un decaimiento hacia el siglo III d.C., presentando una ocupación más reducida y en puntos puntuales durante los ss. IV-V d.C.

\subsubsection{Lancia (Villasabariego, León)}

Se trata de una antigua ciudad astur-romana de gran importancia, que llegó a tener 30.000 habitantes en una extensión de 60 hectáreas, y que en la actualidad está siendo afectada por las obras de la autovía A-60, que unirá León con Valladolid. El yacimiento arqueológico ha sido excavado desde el siglo XIX, pero tales estudios no se han visto acompañados de la adecuada conservación de los restos (VV.AA. 1999). 
Durante la época romana, ss. I-III d.C., la ciudad se reconstruye y crece hasta que se abandona de manera definitiva en el siglo IV d.C., aunque es posible que en las zonas periféricas existiese algún tipo de pervivencia en el lugar.

Todos estos yacimientos leoneses y zamoranos de los que provienen las cuentas, trabajadas y/o sin trabajar, de variscita que hemos analizado tienen un precedente indígena (salvo el campamento de Petauonium) y tras la conquista romana, experimentan un auge durante, principalmente, los ss. I-III d.C., momento a partir del cual se produce un paulatino abandono de los lugares, que continúan siendo ocupados por medio de entidades menores.

\subsection{Las villas romanas bajoimperiales. Los mosaicos}

En primer lugar, hay que tener en cuenta que no todo yacimiento de época romana que se localiza en el campo es una villa. Desconocemos cómo podrían ser la distribución y la jerarquía del poblamiento rural en la Meseta -concentrado (uici, pagi, castella, etc.) o disperso (tuguria, casae, villae, etc.), sin olvidarnos de las instalaciones ligadas a la red viaria (stationes, mansiones, etc.)-(Regueras 2013: 34). Una villa romana, como un cortijo andaluz, es al tiempo vivienda y finca o heredad, donde se diferencia una pars urbana (vivienda), una pars rustica et fructuaria (almacenes, viviendas de colonos, talleres, etc.) y un fundus (donde se distingue el ager, dedicado a los cultivos; el saltus, al pastoreo y ganadería; y la silua o bosque, que aprovisionaría de caza, madera y combustible a la villa).

Hemos podido analizar teselas verdes de cuatro mosaicos, dos palentinos (La Olmeda y Villa Possidica) y dos de León (Quintana del Marco y La Milla del Río) que nos han proporcionado unos datos de gran interés que pasamos a detallar (fig. 2).

\subsubsection{Aquiles en Skyros. La Villa Romana La Olmeda (Pedrosa de la Vega, Palencia)}

El complejo arqueológico La Olmeda se encuentra situada al norte de la provincia de Palencia dentro del término municipal de Pedrosa de la Vega, a $62 \mathrm{~km}$ al norte de la capital y $5 \mathrm{~km}$ al sur de Saldaña, cabecera de comarca, donde se encuentra situado el uicus del Alto de La Morterona, un castro prerromano donde se documenta una secuencia cultural que se extiende desde el Bronce final a la época visigoda, y que presenta una fase coetánea al momento de mayor esplendor de la villa. El centro neurálgico de todo el yacimiento es la gran vivienda señorial de los siglos IV-V d.C., un auténtico palacio, que ocupa una extensión aproximada de $4400 \mathrm{~m}^{2}$. La Olmeda ocupa un lugar destacado en el panorama arqueológico español debido a la existencia de un importante conjunto de mosaicos y a la extraordinaria circunstancia de que los restos fueron protegidos desde su descubrimiento en 1968.

Entre todas las habitaciones destaca el majestuoso salón $\left(175 \mathrm{~m}^{2}\right)$ u oecus (V-14), ámbito donde el señor de la casa o dominus recibiría a familiares y clientes de un modo protocolario y desde donde administraría su vasta propiedad. Aquí es donde se hallan los mosaicos figurados más importantes de la villa palentina. En los bordes, una ancha banda de mosaico geométrico. La parte central la ocupa una escena propia del ciclo troyano, más concretamente de la vida de Aquiles. Se trata del momento exacto del descubrimiento del héroe por Ulises en el gineceo de Lycomedes, rey de Skyros. De esta escena, reproducida en la Antigüedad, se conservan ejemplos en pintura (Pompeya, Domus Aurea, etc.), mosaicos, sarcófagos, etc. perdurando a lo largo de la historia hasta la pintura y escultura contemporáneas. Alrededor de esta escena hay un friso con rostros humanos (se conservan 14 de un total de 18) dispuestos dentro de medallones. Bajo todo ello se sitúa una escena de cacería o, más bien, diversas viñetas cinegéticas contiguas y superpuestas (Abásolo y Martínez 2012: 33-44). La calidad de este mosaico queda patente en su color (más de 40 tonos diferentes) y en el tamaño de las teselas (en ocasiones no superan los dos milímetros).

Para el mosaico de La Olmeda se tomaron un total de once mediciones (las teselas de color verde presentes en el mosaico son incalculables y están presentes en gran parte de los temas desarrollados en el mismo): seis proceden del mosaico de Aquiles en Skyros, tres de los «retratos» que bordean la escena principal, una sobre una de las estaciones (la Primavera) y la última de la figura principal de la escena de caza.

\subsubsection{Océano y las Nereidas. Villa Possidica. Cercado de San Isidro (Dueñas, Palencia)}

Entre 1962 y 1963 salieron a la luz, en la localidad palentina de Dueñas, junto a la Abadía de San IsidroMonasterio de la Trapa, los restos de unos baños pertenecientes a una villa de época tardía, a la que se conoce como "Cercado de San Isidro" o, más comúnmente, "Villa Possidica" (Palol 1963: 6-7; Revilla et al. 1964). 
Entre los hallazgos musivarios que se llevaron a cabo en esas primeras excavaciones destacaron el mosaico de “Océano y las Nereidas", y el de la cabeza de un caballo con una inscripción (Amoris, seguramente de su nombre), de gran calidad pero, desgraciadamente, desaparecido (Palol 1963: 8-9 y 29-32; Revilla et al. 1964: 11-12).

Entre los años 1991-1992 se realizan nuevas actuaciones que tuvieron como objetivo primordial la consolidación de los restos hallados treinta años antes, momento en el que se produjo el levantamiento del mosaico de "Océano y las Nereidas" (Fernández 2012: 321-327). Desde hace relativamente poco tiempo el mosaico se puede ver expuesto en el Museo Arqueológico de Palencia.

El tema de Océano es utilizado con relativa frecuencia dentro de los repertorios musivarios de la Península (Lugo; Elche; Córdoba; Carranque, Toledo; Quintanilla de la Cueza, Palencia; La Milla del Río, León; entre otros muchos ejemplos).

En el mosaico palentino las teselas de color verde se usaron, principalmente, para la barba de Océano y para los objetos de adornos que presentan las dos Nereidas que le acompañan. En total se tomaron cuatro mediciones, en los puntos indicados (barba de Océanos y adornos de Nereidas).

\subsubsection{Hilas y las Ninfas. Los Villares (Quintana del Marco, León)}

Conocido desde antiguo (las primeras actuaciones tienen lugar en 1898), el yacimiento está fuertemente alterado tanto por la rebusca indiscriminada, como por el laboreo agrícola. Además, ha sido objeto de excavaciones puntuales, conociéndose una mínima parte de lo que debió ser la parte noble de la villa. Uno de estos hallazgos es el mosaico de "Hilas y las Ninfas", el mosaico teselado más famoso de la provincia de León, que se conserva, tras una serie de trabajos de restauración, en el Museo Arqueológico de dicha provincia, ocupando un lugar de privilegio dentro de su exposición permanente (Regueras et al. 1994).

Se trata un tema mitológico recogido ampliamente en la literatura clásica y en la musivaria de época tardorromana (Carranque, Volubilis, etc.): El rapto de Hilas por parte de las ninfas.

En el mosaico leonés se tomaron un total de seis mediciones en los puntos donde se constataba la presencia de teselas de color verde. De este modo fueron objeto de análisis la punta de la lanza de Hilas, los mantos de ambas ninfas y la corriente de agua que se sitúa a los pies de la escena.

\subsubsection{Océano (La Milla del Río, León)}

La Milla del Río es una villa romana de la provincia de León de la que se conoce muy poco. Se ha llevado a cabo un número reducido de intervenciones y los hallazgos resultantes han sido muy pocos, destacando el mosaico de Océano que se conserva, al igual que el anterior mosaico, en el Museo Arqueológico de León. Se llevaron a cabo tres lecturas en dicho mosaico en los puntos donde se apreciaban teselas verdes.

\subsubsection{Otros restos}

Al margen de los mosaicos citados, en el museo de León se conservan una serie de ejemplos de núcleos de variscita, tanto de fragmentos en bruto como de planchas ya preparadas para la extracción, suponemos, de teselas verdes. Los mismos fueron recuperados superficialmente en los terrenos de la villa romana de Los Villares, donde se localiza el mosaico de "Hilas y las Ninfas", y en Lancia (Villasabariego, León).

\section{ANÁLISIS DE COMPOSICIÓN}

A la hora de establecer la procedencia de las variscita seguiremos los protocolos analíticos descritos por Odriozola et al. (2010) que relacionan el cociente atómico $\mathrm{P} / \mathrm{Al}$ con la paragénesis de la roca para así establecer el origen del mineral.

Fluorescencia de rayos $X$ : Las muestras de variscita fueron analizadas mediante procedimientos no destructivos con una fluorescencia de rayos $\mathrm{X}$ portátil Oxford Instrument XMET-7500. El equipo monta un tubo de rayos X Eclipse E4LE con ánodo de $\mathrm{Rh}$, un detector Silicon Drift Detector (SDD) y un cambiador automático de 5 filtros. Los análisis han sido realizados ajustando el tubo a $45 \mathrm{kV}$ y $40 \mu \mathrm{A}$ con una duración de $60 \mathrm{~s}$.

Dada la ausencia de aluminofosfatos certificados para realizar una calibración empírica (Criss y Birks 1968), la cuantificación se ha realizado utilizando el programa calibrado de fábrica SOILS LE que está basada en el método de parámetros fundamentales. Se ha demostrado que el método de parámetros fundamentales es el más adecuado para calcular la composición química cuando no existe un método basado en patrones primarios o cuando se quiere analizar un gran número de elementos (Beckhoff et al. 2006: 403) como es nuestro caso. Recientemente Elam et al. (2004) han demostrado que utilizando parámetros atómicos actualizados se alcanzan errores c. $1 \%$ para los 

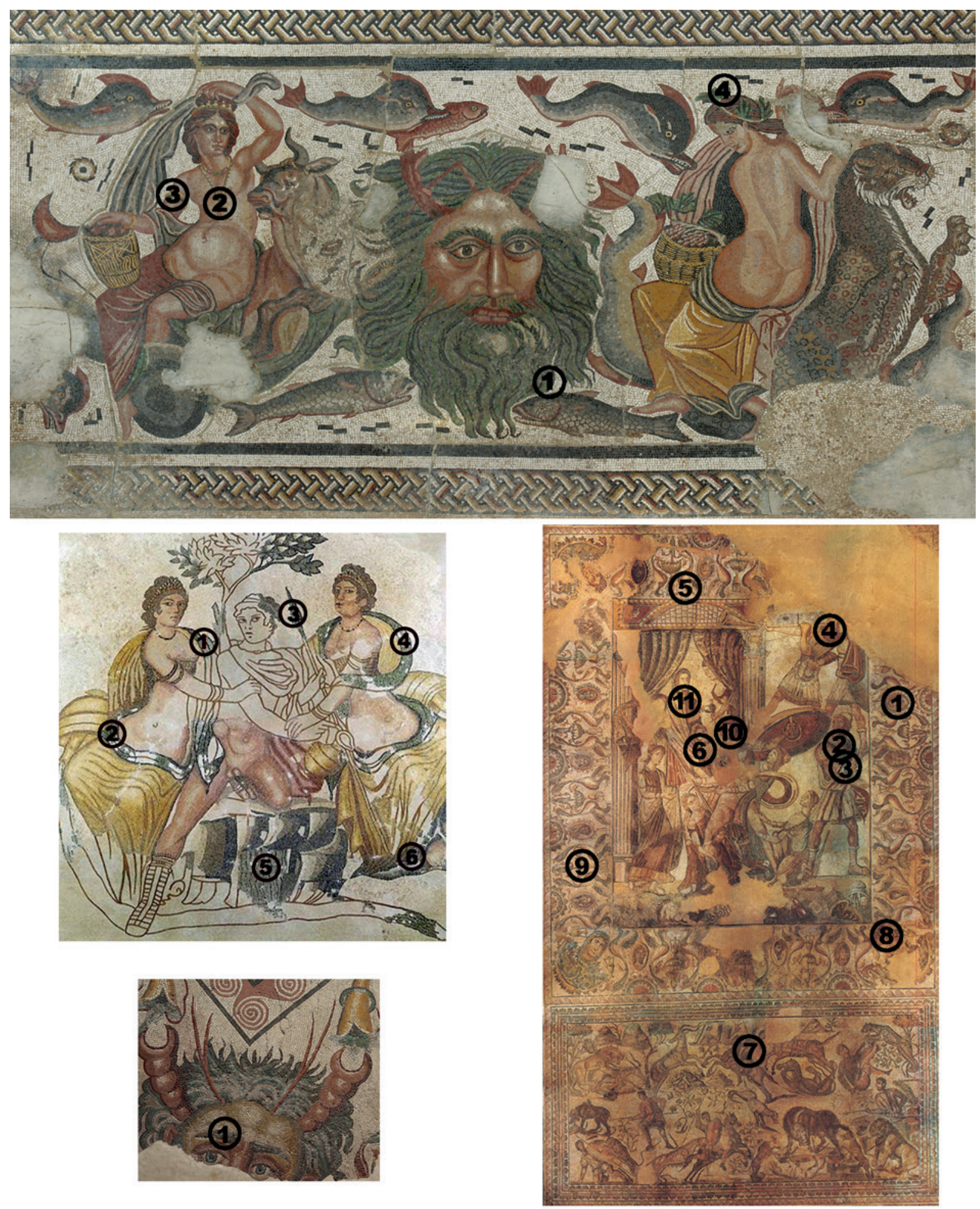

Figura 2. Teselas analizadas en los mosaicos bajoimperiales. Arriba, Océano y las Nereidas (Villa Possidica, Dueñas, Palencia); abajo derecha, Mosaico del Oecus (Villa La Olmeda, Pedrosa de la Vega, Palencia); centro izquierda, Hilas y las Ninfas (Villa Los Villares, Quintana del Marco, León); abajo izquierda, Océano (Villa Milla del Río, León). 
Tabla 1. Distribución de los valores medios medidos para el cociente $\mathrm{P} / \mathrm{Al} \%$ atómico en la principales fuentes y los yacimientos romanos a estudio de la Meseta Norte $(*$ los datos de Can Tintorer proceden de las tablas publicadas por Edo et alii 1998, ** hemos excluido los datos de los análisis Hilas 1 e Hilas 5 de Los Villares, aberrantes posiblemente debido al reducido tamaño de las teselas).

\begin{tabular}{|c|c|c|c|c|c|c|c|c|c|c|}
\hline & $\begin{array}{c}\text { Pico } \\
\text { Centeno }\end{array}$ & $\begin{array}{c}\text { Palazuelo de } \\
\text { las Cuevas }\end{array}$ & $\begin{array}{c}\text { Can } \\
\text { Tintorer* }\end{array}$ & Astorga & $\begin{array}{c}\text { D. } \\
\text { Misleo }\end{array}$ & Castrico & Castro & Lancia & $\begin{array}{c}\text { V. } \\
\text { Villares** }\end{array}$ & $\begin{array}{c}\text { V. } \\
\text { Possidica }\end{array}$ \\
\hline $\begin{array}{c}\text { Media P/A } \\
1 \% \text { atómico }\end{array}$ & 1.74 & 1.21 & 1.04 & 1.34 & 1.19 & 1.27 & 1.37 & 1.37 & 1.22 & 1.21 \\
\hline $\begin{array}{c}\text { Desviación } \\
\text { estándar }\end{array}$ & 0.04 & 0.06 & 0.16 & 0.07 & 0.10 & 0.06 & 0.10 & 0.10 & - & 0.05 \\
\hline $\begin{array}{c}95 \% \text { superior } \\
\text { media }\end{array}$ & 1.75 & 1.22 & 0.98 & 1.51 & 1.43 & 1.34 & 1.53 & 1.53 & - & 1.35 \\
\hline $\begin{array}{c}95 \% \text { inferior } \\
\text { media }\end{array}$ & 1.72 & 1.20 & 1.10 & 1.18 & 0.95 & 1.20 & 1.21 & 1.21 & - & 1.08 \\
\hline $\mathrm{N}$ & 38 & 153 & 29 & 3 & 3 & 5 & 4 & 4 & 1 & 2 \\
\hline
\end{tabular}

elementos mayoritarios (excepto para el $\mathrm{Na}$ y el $\mathrm{Si}$ ) en materiales certificados. Dichos parámetros atómicos actualizados han sido tenidos en cuenta en el programa de calibrado SOILS LE que utilizamos. La cuantificación del porcentaje en peso de elemento se ha realizado asumiendo una matriz de óxidos. A falta de análisis mineralógicos (e.g. Difracción de rayos $\mathrm{X}$ ) asumimos que aquellos materiales de color verde y brillo céreo cuya composición mayoritaria se funda en una relación Fósforo-Aluminio c. 1.0-1.4 se hallarían elaborados en variscita $\left(\mathrm{AlPO}_{4} \cdot 2 \mathrm{H}_{2} \mathrm{O}\right)$. Nótese que en los resultados de las teselas los niveles de Si son más altos como consecuencia de que, debido a la realización de los análisis in situ, la medida incluyó también el mortero, compuesto fundamentalmente por arena, un silicato.

$\mathrm{Si}$ asumimos la hipótesis de que las variaciones en el cociente $\mathrm{P} / \mathrm{Al}$ se deben a la paragénesis del mineral y que, por tanto, este parámetro es capaz de discriminar entre afloramientos (Odriozola et al. 2010) y dado que su variabilidad natural en la fuente es menor que la variabilidad entre fuentes, la coincidencia entre el cociente $\mathrm{P} / \mathrm{Al}$ del producto y fuente será un indicador fiable del origen del producto. En la tabla 1 podemos observar cómo los valores medios de las fuentes se encuentran bien definidos y distantes y cómo los valores de las cuentas coinciden con los valores de las minas de Palazuelo de las Cuevas (fig. 3). Asimismo, todas las teselas de color verde que resultaron ser variscita (Océano y las Nereidas, Dueñas, Palencia e Hilas y las Ninfas, Quintana del Marco, León) presentan un cociente cuyos valores vuelven a coincidir con los de las minas localizadas en Palazuelos de las Cuevas.
Mientras que en la tabla 2 podemos observar la composición elemental de los materiales estudiados.

\section{Resultados}

A lo largo del trabajo hemos visto la procedencia de las diferentes cuentas y teselas analizadas y, de una forma somera, los yacimientos donde se encuentran o donde están localizadas.

Sobre las cuentas de collar, resultaron ser de variscita la gran mayoría de las piezas analizadas, salvo las dos recuperadas en Petauonium. En cuanto a las teselas de color verde analizadas podemos decir que en dos de los mosaicos (Océano y Las Nereidas e Hilas y las Ninfas, en el cual no todas eran variscita) resultaron ser variscita, mientras que en los otros dos (Aquiles en Skyros y Océano) los análisis sugieren un silicato indeterminado. En ambos casos, objetos de adorno y teselas de variscita, la procedencia de este mineral verde es la misma, Palazuelo de las Cuevas.

\section{CONCLUSIONES}

Visto todo lo anterior llegamos a la conclusión, en primer lugar, que durante el Alto Imperio el uso de la variscita se restringe al ámbito de la glyptica. Durante el Bajo Imperio este mineral se deja de explotar de una manera continuada y sistemática siendo sustituido, en la mayoría de los casos, para la elaboración de los objetos de adorno por la pasta vítrea. Aun así hay ciertos 


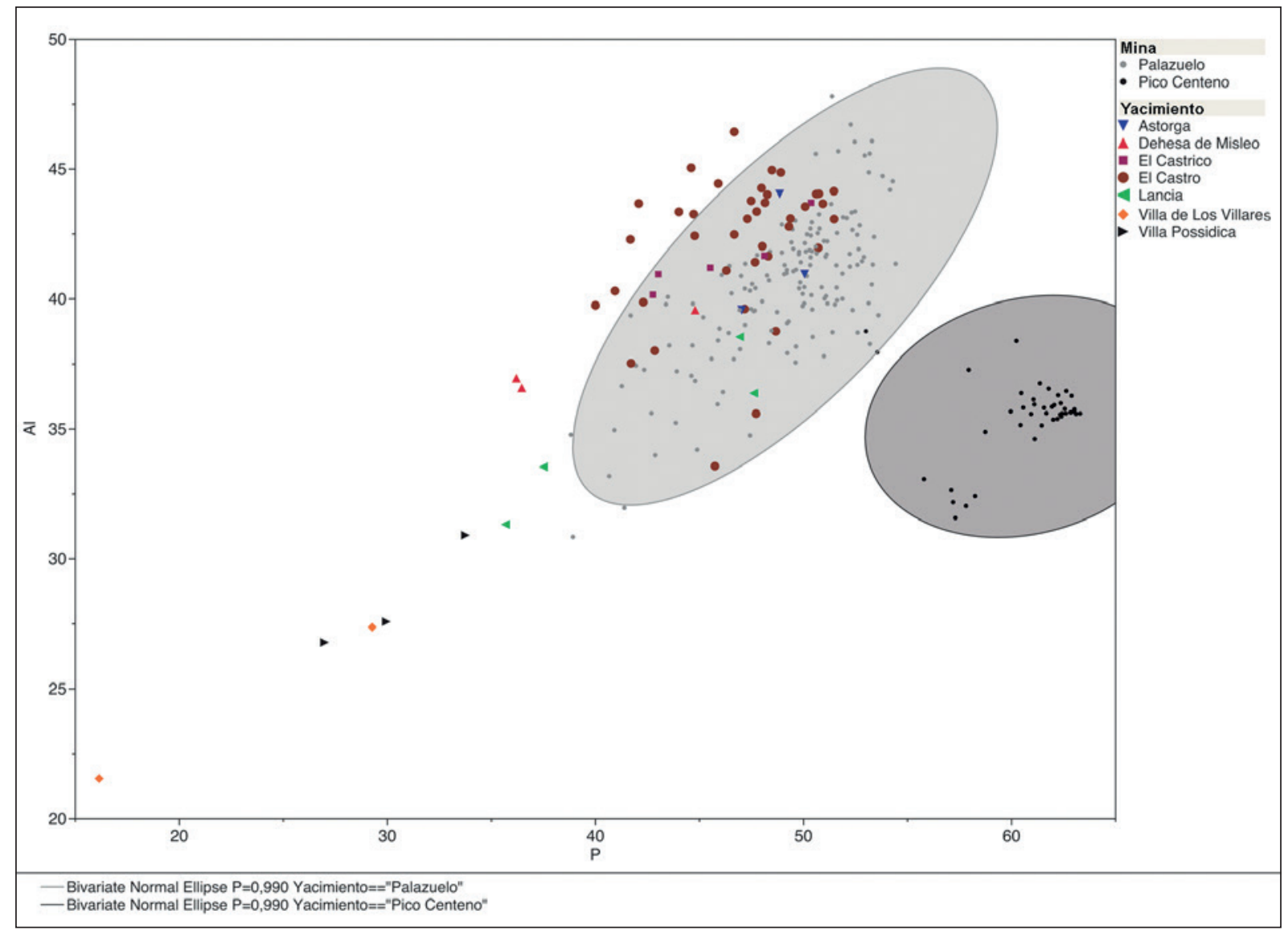

Figura 3. Gráfica de la relación $\mathrm{P} / \mathrm{Al}$ de los diversos materiales romanos de variscita analizados en comparación con la proporción de la variscita de las minas de Palazuelo de las Cuevas (Zamora) y Pico Centeno (Huelva).

musivarios/artesanos que conocen la existencia de esta "piedra" de color verde y recurren a ella para la elaboración de las teselas de dicho color para realizar los mosaicos, pero de una manera residual (fig. 4).

Una vez vistas todas las analíticas realizadas y las características de los yacimientos romanos donde se han encontrado las piezas analizadas, podemos diferenciar dos fases o etapas en la utilización de la variscita durante la época romana, que vienen a coincidir, a rasgos generales, con los dos períodos de máxima importancia: 1. Una primera fase, altoimperial, donde la variscita se utilizaría para la realización de objetos de adorno (Astorga, Lancia, etc.). Habría que situarla durante los ss. I-III d.C. Los artesanos se aprovisionarían de las minas más cercanas, las cuales serían conocidas y estarían en uso presentando una explotación, más o menos, intensa. Todo ello haría de los objetos de variscita un producto de lujo de bajo coste, del gusto de los consumidores, y que podría ser adquirido por un amplio margen de la población.
2. Una segunda fase, bajoimperial, momento en el cual la variscita parece que se deja de utilizar como objeto de adorno y pasa a ser usada en la elaboración de teselas de color verde para la realización de los grandes mosaicos que adornan las villas tardías, pero de una forma residual. Habría que fecharla durante los ss. IV-V d.C. En estos momentos las minas recibirían "visitas" ocasionales por parte de los musivarios (no todos, sólo los que conociesen la existencia de esta piedra de color verde) para recoger el mineral en bruto que, posteriormente, sería trabajado.

Este cambio se puede deber a la caída de la demanda interna de objetos de adorno en las ciudades debido a la crisis del s. III y el abandono, por parte de los "ricos" propietarios, de las mismas. Sin olvidarnos de un paulatino abandono en la explotación de las minas de variscita, como consecuencia de la caída de las ventas de estos productos por la inestabilidad existente en la época. 


\begin{tabular}{|c|c|c|c|c|c|c|c|c|c|c|c|c|c|c|c|c|c|c|c|c|c|}
\hline $\bar{N}$ & $\begin{array}{l}\circ \\
\stackrel{0}{\circ}\end{array}$ & 各 & & $\stackrel{\text { ô }}{\circ}$ & $\stackrel{0}{\circ}$ & 总 & & 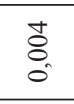 & $\begin{array}{l} \pm \\
0 \\
0\end{array}$ & 灾 & $\stackrel{\text { on }}{0}$ & & $\begin{array}{l} \pm \\
0 \\
0 \\
0^{\circ}\end{array}$ & 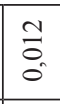 & $\begin{array}{l}\bar{z} \\
0_{0}\end{array}$ & & $\begin{array}{l}0 \\
0 \\
0\end{array}$ & $\begin{array}{l}0 \\
0 \\
0\end{array}$ & $\begin{array}{l}\stackrel{0}{\circ} \\
\stackrel{0}{0}\end{array}$ & $\begin{array}{l}\overrightarrow{0} \\
0 \\
0\end{array}$ & 离 \\
\hline$\check{\check{n}}$ & $\tilde{o}_{0}^{\circ}$ & & $\begin{array}{l}\text { on } \\
\vdots\end{array}$ & & $\begin{array}{l}\text { on } \\
\vdots\end{array}$ & & & & & & \&े & & & & & & & & : & & \\
\hline 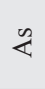 & $\begin{array}{l}\tilde{O} \\
\delta^{\circ}\end{array}$ & ठิ & 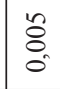 & 苛 & $\begin{array}{l}0 \\
0 \\
0\end{array}$ & $\begin{array}{l}\text { gै } \\
0 \\
0\end{array}$ & $\hat{\tilde{o}}$ & ठे & oे & $\begin{array}{l}0 \\
0 \\
0 \\
0\end{array}$ & $\begin{array}{l}0 \\
\text { O্. } \\
0\end{array}$ & $\begin{array}{l}0 \\
\stackrel{0}{0} \\
0\end{array}$ & & $\begin{array}{l}0 \\
\stackrel{0}{0}\end{array}$ & 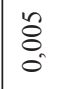 & $\tilde{o}_{0}^{\circ}$ & 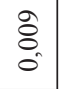 & 号 & $\overline{\sigma_{0}}$ & $\begin{array}{l}\hat{\sigma} \\
0_{0}^{\circ}\end{array}$ & 音 \\
\hline ธ & & & & & & & & & & & & & & & ठ̊ & & ठ̊. & & & & \\
\hline J & & & & & & & & & & & $\stackrel{\delta}{0}$ & & & & & $\begin{array}{l}\overrightarrow{0} \\
\dot{0}\end{array}$ & & & & & \\
\hline 诖 & is & $\begin{array}{l}3 \\
i\end{array}$ & 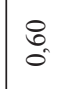 & $\begin{array}{l} \pm \\
\text { in } \\
\text { in }\end{array}$ & $\hat{n}$ & $\stackrel{0}{=}$ & $\stackrel{m}{3}$ & 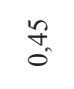 & $\tilde{0}$ & $\hat{n}$ & $\underset{i}{\mathbb{i}}$ & $\underset{-}{\mathbb{G}}$ & fos & $\begin{array}{l}\infty \\
0 \\
0\end{array}$ & 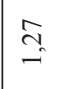 & $\begin{array}{l}\vec{n} \\
i\end{array}$ & 8 & $\begin{array}{l}\infty \\
\circ\end{array}$ & $\begin{array}{l}\stackrel{\infty}{0} \\
\stackrel{0}{=}\end{array}$ & $\stackrel{0}{0}$ & $\begin{array}{l}0 \\
n \\
0\end{array}$ \\
\hline ¿゙ & के & if & $\begin{array}{l}\bar{\infty} \\
0_{0}^{\prime}\end{array}$ & त̂ & $\begin{array}{l}0 \\
0 \\
0 \\
0\end{array}$ & $\begin{array}{l}\stackrel{+}{d} \\
\stackrel{0}{0}\end{array}$ & m. & $\hat{n}$ & $\hat{m}$ & fo & $\begin{array}{l}\text { î } \\
\text { on }\end{array}$ & $\stackrel{\infty}{0}_{0}^{0}$ & $\tilde{O}$ & ป̊ & $\underset{0}{\sharp}$ & $\stackrel{m}{\tilde{0}}$ & $\vec{F}$ & $\begin{array}{c}\hat{m} \\
0\end{array}$ & $:$ & $\hat{n}$ & त् \\
\hline$>$ & $\begin{array}{l}5 \\
0 \\
0\end{array}$ & & $\frac{\circ}{0}$ & $\because$ & $\begin{array}{l}\hat{0} \\
0\end{array}$ & & $\begin{array}{l}\infty \\
0 \\
0\end{array}$ & & $\bar{r}$ & & & $\stackrel{\infty}{\infty}$ & $\begin{array}{l}\infty \\
0 \\
0\end{array}$ & $\because$ & $\stackrel{0}{0}$ & तี & & $\hat{\sigma}_{0}$ & ${ }_{0}^{t}$ & & \\
\hline$F$ & 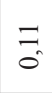 & $\underset{\delta}{\tilde{\sigma}}$ & $\mathbb{0}_{0}^{t}$ & $\overline{\tilde{\sigma}}$ & $\begin{array}{l}0 \\
\tilde{c}_{0}\end{array}$ & $\stackrel{\simeq}{0}$ & $\stackrel{7}{0}$ & $\begin{array}{l} \pm \\
0 \\
0\end{array}$ & ì & $\stackrel{0}{0}$ & $=$ & $\stackrel{n}{0}$ & $\begin{array}{l}0 \\
0\end{array}$ & $\stackrel{\Rightarrow}{0}$ & $\stackrel{ \pm}{ \pm}$ & $\hat{o}_{0}$ & $\overrightarrow{=}$ & $\stackrel{n}{0}$ & $\begin{array}{l}\tilde{y} \\
\tilde{0}\end{array}$ & $\stackrel{m}{0}$ & $\stackrel{0}{0}$ \\
\hline$\tilde{0}$ & of & $\because$ & $\because$ & $\begin{array}{l}1 \\
0 \\
0\end{array}$ & $\stackrel{0}{0}$ & & & $\stackrel{n}{0}$ & $\frac{J}{0}$ & & $\stackrel{\infty}{0}$ & 8 & $\stackrel{\sim}{\circ}$ & $\begin{array}{l}n \\
0 \\
0\end{array}$ & o. & $\stackrel{\infty}{0}$ & $\begin{array}{l}\infty \\
0 \\
0\end{array}$ & $\hat{O}_{0}$ & $\frac{0}{0}$ & ${ }_{0}^{t}$ & \\
\hline$\triangle$ & & & & & & & & & & & $\begin{array}{l}\infty \\
\infty \\
0\end{array}$ & $\begin{array}{l}\hat{n} \\
\hat{0}\end{array}$ & है & & & & & & & & \\
\hline $\bar{U}$ & $\begin{array}{l}\Delta \\
\text { a }\end{array}$ & $\stackrel{2}{=}$ & $\stackrel{\vec{b}}{-}$ & $\frac{t}{0}$ & $\begin{array}{l}\bar{\infty} \\
0 \\
0\end{array}$ & $\stackrel{\sim}{\longrightarrow}$ & $\stackrel{0}{=}$ & $\tilde{n}$ & $\stackrel{+}{\underset{\sim}{-}}$ & $\vec{F}$ & $\stackrel{\vec{i}}{ }$ & $\begin{array}{l}\vec{t} \\
\text { ì }\end{array}$ & $\hat{m}$ & $\begin{array}{l}2 \\
\infty \\
0\end{array}$ & $\stackrel{n}{-}$ & 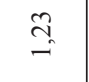 & $\stackrel{m}{-}$ & $\stackrel{+}{\sim}$ & $\hat{o}_{-}$ & $\stackrel{n}{\rightarrow}$ & $\stackrel{\infty}{\stackrel{\infty}{-}}$ \\
\hline$n$ & $\frac{7}{0}$ & तี & 足 & $\begin{array}{l}0 \\
0 \\
0\end{array}$ & $\begin{array}{l}0 \\
0 \\
0\end{array}$ & & $\stackrel{\circ}{0}$ & $\stackrel{m}{0}$ & $\frac{\infty}{0}$ & $\overrightarrow{\tilde{\sigma}}$ & $\hat{n}$ & 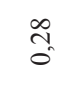 & $\stackrel{n}{i}$ & $\stackrel{m}{0}$ & તુ & $\Rightarrow$ & $\begin{array}{l}0 \\
0\end{array}$ & $\frac{\partial}{0}$ & 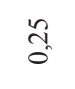 & $\Rightarrow$ & $\stackrel{m}{0}$ \\
\hline$a$ & $\begin{array}{l}\vec{Y} \\
\vec{f}\end{array}$ & 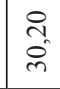 & $\begin{array}{l}\text { กิ } \\
\text { ลे }\end{array}$ & $\begin{array}{l}\infty \\
\vec{m}\end{array}$ & $\begin{array}{l}\overrightarrow{\infty_{n}} \\
\vec{m}\end{array}$ & $\begin{array}{l}\text { ले } \\
\text { ले }\end{array}$ & $\begin{array}{l}\hat{\alpha} \\
\text { oे }\end{array}$ & $\begin{array}{c}\underset{m}{2} \\
\text { n}\end{array}$ & $\begin{array}{l}\infty \\
0 \\
\text { i }\end{array}$ & $\begin{array}{l}\stackrel{\infty}{\sim} \\
\tilde{f}\end{array}$ & $\begin{array}{l}R \\
\text { si }\end{array}$ & $\begin{array}{l}\delta \\
\dot{m}\end{array}$ & $\overrightarrow{\widehat{i}}$ & $\begin{array}{l}\text { fo } \\
\stackrel{8}{8}\end{array}$ & $\begin{array}{l}n \\
0 \\
\tilde{m}\end{array}$ & $\overrightarrow{\hat{n}^{\circ}}$ & $\begin{array}{l}\circ \\
\hat{m}\end{array}$ & $\stackrel{0}{m}$ & $\stackrel{\vec{\sim}}{\overrightarrow{0}}$ & $\begin{array}{l}\vec{\sigma} \\
\vec{m}\end{array}$ & $\begin{array}{l}\text { ते } \\
\text { ลे }\end{array}$ \\
\hline$\vec{n}$ & $\stackrel{n}{n}$ & $\stackrel{\ominus}{\stackrel{f}{*}}$ & $\begin{array}{l}\stackrel{n}{f} \\
\leftarrow\end{array}$ & $\begin{array}{c}0 \\
\text { m } \\
+\end{array}$ & $\begin{array}{l}\stackrel{े}{\sigma} \\
\text { o }\end{array}$ & $\stackrel{0}{\sim}$ & id & $\underset{i}{i}$ & $\stackrel{\sim}{\sigma}$ & $\underset{\Xi}{-}$ & $\begin{array}{l}\vec{n} \\
i n\end{array}$ & $\stackrel{m}{m}$ & $\begin{array}{c}\vec{c} \\
\dot{m}\end{array}$ & $\begin{array}{l}f \\
\text { rn }\end{array}$ & तु & $\begin{array}{l}n \\
0 \\
i n\end{array}$ & $\stackrel{\circ}{\rightarrow}$ & $\underset{i}{\tilde{i}}$ & $\begin{array}{l}n \\
\tilde{\sigma} \\
\end{array}$ & $\overrightarrow{\vec{\sigma}}$ & $\underset{f}{\stackrel{f}{*}}$ \\
\hline « & $\begin{array}{l}5 \\
\stackrel{+}{+}\end{array}$ & $\begin{array}{l}0 \\
\tilde{m} \\
\tilde{i}\end{array}$ & $\begin{array}{l}\vec{b} \\
\dot{d}\end{array}$ & $\begin{array}{l}8 \\
0 \\
0\end{array}$ & $\begin{array}{l}1 \\
0 \\
\text { i }\end{array}$ & $\begin{array}{l}\overrightarrow{0} \\
\text { i }\end{array}$ & $\begin{array}{l}\mathbf{D} \\
\text { s. } \\
0\end{array}$ & $\begin{array}{l}\text { oे } \\
\text { in }\end{array}$ & $\begin{array}{l}\stackrel{0}{ \pm} \\
\stackrel{d}{d}\end{array}$ & $\begin{array}{l}\infty \\
2 \\
\text { nิ }\end{array}$ & $\begin{array}{l}8 \\
\text { i } \\
\text { i }\end{array}$ & $\begin{array}{l}8 \\
\stackrel{0}{+}\end{array}$ & $\begin{array}{l}n \\
n \\
+ \\
+\end{array}$ & $\stackrel{\hat{a}}{2}$ & $\begin{array}{l}2 \\
\dot{d} \\
\text { d }\end{array}$ & $\begin{array}{l}\text { fa } \\
\text { i }\end{array}$ & $\begin{array}{l}\text { nे } \\
\text { ते }\end{array}$ & $\begin{array}{l}\text { ż } \\
\text { ป̂. }\end{array}$ & $\begin{array}{l}\overrightarrow{0} \\
\stackrel{\infty}{\rightarrow}\end{array}$ & 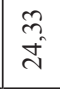 & $\begin{array}{l}\text { ㄱ. } \\
\text { in }\end{array}$ \\
\hline 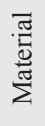 & 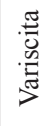 & 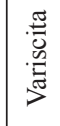 & 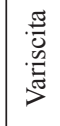 & 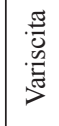 & 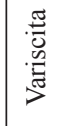 & 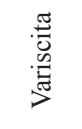 & 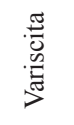 & 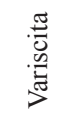 & 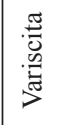 & 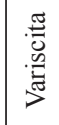 & 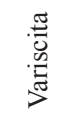 & 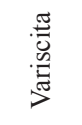 & 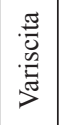 & 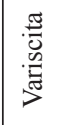 & 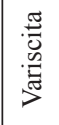 & 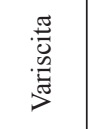 & 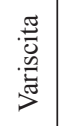 & 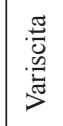 & 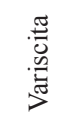 & 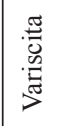 & 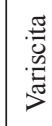 \\
\hline 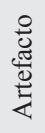 & 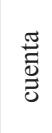 & 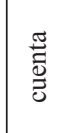 & 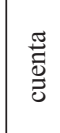 & 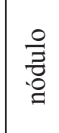 & 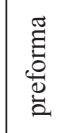 & 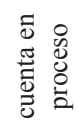 & 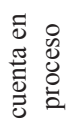 & 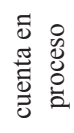 & 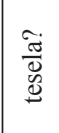 & 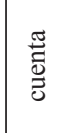 & 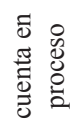 & 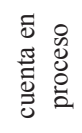 & 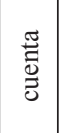 & 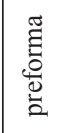 & 芯 & 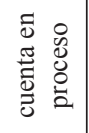 & 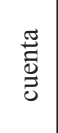 & $\begin{array}{l}\text { 萼 } \\
\overline{\mathrm{z}}\end{array}$ & 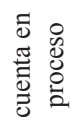 & 莺 & 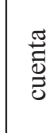 \\
\hline 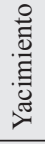 & 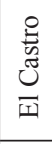 & 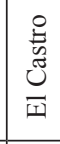 & 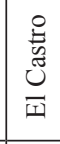 & 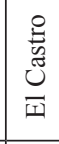 & 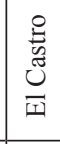 & 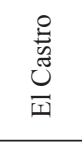 & 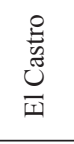 & 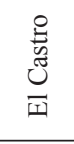 & 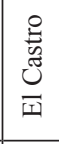 & 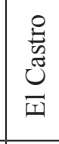 & 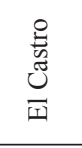 & 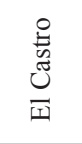 & 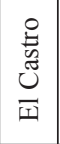 & 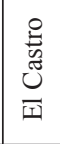 & 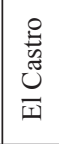 & 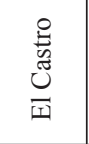 & 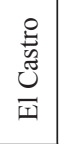 & 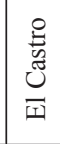 & 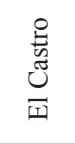 & 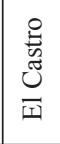 & 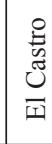 \\
\hline 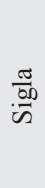 & 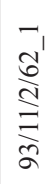 & 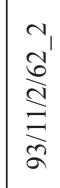 & 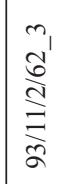 & 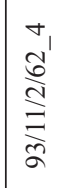 & 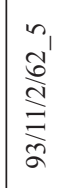 & 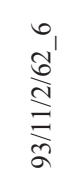 & 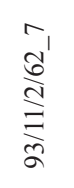 & 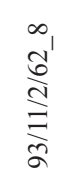 & 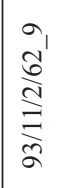 & 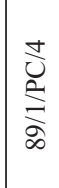 & $\begin{array}{l}\stackrel{n}{0} \\
\stackrel{\Xi}{L} \\
\stackrel{\infty}{L}\end{array}$ & 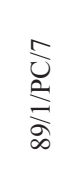 & 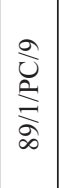 & 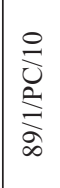 & 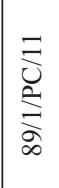 & 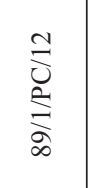 & 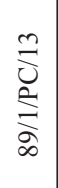 & 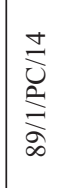 & $\frac{n}{\vdots}$ & $\begin{array}{l}0 \\
\frac{1}{0} \\
\stackrel{2}{2} \\
\stackrel{\infty}{\infty}\end{array}$ & 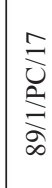 \\
\hline
\end{tabular}




\begin{tabular}{|c|c|c|c|c|c|c|c|c|c|c|c|c|c|c|c|c|c|c|c|c|c|c|c|}
\hline$\grave{N}$ & : & 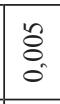 & 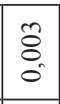 & $\stackrel{0}{0}$ & $\overline{\mathrm{z}}$ & ठิ & & & $\begin{array}{l}\text { ठै } \\
0^{\circ}\end{array}$ & 号 & $\stackrel{8}{\circ}$ & $\begin{array}{l}\text { do } \\
0 \\
0\end{array}$ & $\stackrel{\Delta}{O_{0}^{\circ}}$ & $\begin{array}{l}\text { ते } \\
\text { o. }\end{array}$ & $\stackrel{0}{\circ}$ & & $\begin{array}{l}0 \\
0 \\
0 \\
0\end{array}$ & $\begin{array}{c}\tilde{\delta} \\
0 \\
0\end{array}$ & & $\stackrel{m}{0}$ & $\tilde{o}_{0}$ & & $\stackrel{I}{0}$ \\
\hline 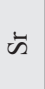 & $\tilde{o}_{0}$ & $\stackrel{0}{0}$ & ठ̊. & & $\begin{array}{l}\text { पे } \\
\stackrel{0}{0}\end{array}$ & $\begin{array}{l}+ \\
\stackrel{0}{0} \\
0\end{array}$ & & & & & $\begin{array}{l}\text { oे } \\
\text { o }\end{array}$ & $\stackrel{\infty}{\circ}$ & & $\stackrel{\bar{\sigma}}{0}$ & 总 & & $\tilde{o}_{0}^{\circ}$ & & & & $\begin{array}{l}\infty \\
\tilde{O}_{0}^{0}\end{array}$ & $\begin{array}{l}\text { to } \\
0_{0}^{\prime}\end{array}$ & \\
\hline 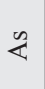 & & & & $\stackrel{\partial}{\overrightarrow{0}}$ & $\begin{array}{l} \\
\text { İ } \\
0\end{array}$ & \& & $\stackrel{\circ}{\circ}$ & 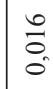 & & $\stackrel{m}{0}$ & $\frac{\Delta}{0}$ & 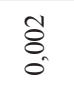 & & ${ }_{0}^{n}$ & 范 & $\begin{array}{l}\tilde{O} \\
\stackrel{0}{0}\end{array}$ & & $\begin{array}{l}\text { ¿ें } \\
\text { o. }\end{array}$ & 妾 & $\begin{array}{l}\overrightarrow{\sigma_{0}} \\
0^{\circ}\end{array}$ & $\stackrel{+}{\overrightarrow{0}}$ & $\begin{array}{l}\infty \\
: \\
0\end{array}$ & $\begin{array}{l}\text { : } \\
\text { : }\end{array}$ \\
\hline ี & & & & & & $\begin{array}{l}\text { ठे } \\
\text { ¿ }\end{array}$ & & & $\stackrel{\text { ô }}{\circ}$ & & & & & & & & $\begin{array}{l} \pm \\
\stackrel{0}{0} \\
0\end{array}$ & & & & 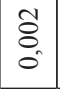 & $\begin{array}{l}\text { ô } \\
\dot{0}\end{array}$ & \\
\hline$\Xi$ & & & & & & & & & & & & & & $\stackrel{\vec{\sigma}}{0}$ & & & $\bar{\sigma}$ & & $\begin{array}{l}\vec{\sigma} \\
\dot{\sigma}_{0}\end{array}$ & $\begin{array}{l}\overrightarrow{0} \\
\dot{0}\end{array}$ & & : & \\
\hline II & $\exists$ & $\mid \begin{array}{l}0 \\
\infty \\
0 \\
0\end{array}$ & $\begin{array}{l}0 \\
n \\
0 \\
0\end{array}$ & $\stackrel{\sim}{\sim}$ & $\vec{b}_{0}$ & $\underset{0}{\infty}$ & $\vec{F}_{0}$ & $\overbrace{0}^{t}$ & f̧ & 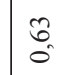 & ले & $\begin{array}{c}\hat{\infty} \\
0 \\
0\end{array}$ & : & $\overrightarrow{0}$ & $\stackrel{m}{\rightarrow}$ & $\stackrel{n}{f}$ & $\stackrel{m}{\stackrel{m}{i}}$ & $\begin{array}{l}\hat{i} \\
i\end{array}$ & $\underset{\infty}{-\infty}$ & $\begin{array}{l}\tilde{b} \\
i\end{array}$ & $\overrightarrow{m_{1}}$ & $\overrightarrow{0}$ & के \\
\hline む゙ & हे & $\stackrel{0}{0}$ & $\begin{array}{l}\text { ते } \\
\text { - }\end{array}$ & $\begin{array}{l}\text { I. } \\
\text { ó }\end{array}$ & $\begin{array}{l}0 \\
0\end{array}$ & fó & $\hat{\tilde{c}}$ & ते & ల్ర & ठे & $\stackrel{\infty}{0}$ & oे & $\stackrel{?}{\circ}$ & $\stackrel{8}{\circ}$ & $\tilde{n}$ & 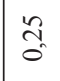 & $\begin{array}{c}0 \\
\tilde{m}_{0} \\
0\end{array}$ & $\hat{m}_{0}$ & 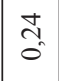 & $\hat{n}$ & $\begin{array}{l}0 \\
\vdots \\
0\end{array}$ & ले & in \\
\hline$>$ & $\stackrel{0}{\stackrel{0}{0}}$ & $\frac{ \pm}{0}$ & $\ddot{\theta}_{0}$ & & to & oे & $\stackrel{2}{0}$ & $\begin{array}{l} \pm \\
0\end{array}$ & $\stackrel{8}{\circ}$ & $\stackrel{8}{\circ}$ & $\stackrel{8}{0}$ & $\stackrel{0}{\circ}$ & & $\stackrel{7}{0}$ & $\stackrel{0}{\circ}$ & & & $\stackrel{0}{\circ}$ & $\begin{array}{l}0 \\
0 \\
0\end{array}$ & $\stackrel{\circ}{\circ}$ & & $\Rightarrow$ & : \\
\hline$F$ & $\stackrel{m}{0}$ & $\stackrel{\Rightarrow}{0}$ & $\begin{array}{l}\vec{y} \\
\tilde{0}\end{array}$ & $\stackrel{7}{0}$ & $\stackrel{\circ}{\circ}$ & $\stackrel{\circ}{\circ}$ & oे & : & : & $\frac{7}{0}$ & $\stackrel{8}{\circ}$ & $\stackrel{n}{0}$ & $\frac{0}{0}$ & ה̃ & $\frac{n}{0}$ & $\stackrel{\pi}{\circ}$ & $\begin{array}{c}\hat{2} \\
\hat{0}\end{array}$ & $\cong$ & $\begin{array}{l}\infty \\
0 \\
0\end{array}$ & n. & $\stackrel{m}{0}$ & $\stackrel{8}{0}$ & $\stackrel{m}{0}$ \\
\hline ש & $\stackrel{\circ}{\circ}$ & $\stackrel{8}{8}$ & $\stackrel{8}{\circ}$ & $\ddot{0}$ & : & $\stackrel{\Delta}{0}$ & $\stackrel{+}{0}$ & & $\stackrel{\infty}{\circ}$ & $a_{0}^{0}$ & $\begin{array}{l}\infty \\
0 \\
0\end{array}$ & $\frac{ \pm}{0}$ & $\stackrel{8}{0}$ & $\stackrel{8}{\circ}$ & $\hat{0}$ & $\stackrel{0}{0}$ & $\begin{array}{l}0 \\
0\end{array}$ & $\Rightarrow$ & $\frac{0}{0}$ & $\frac{0}{0}$ & $\hat{n}$ & $\stackrel{2}{0}$ & I \\
\hline 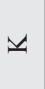 & $\hat{n}$ & ก. & & & & & & & & & $\stackrel{0}{n}$ & $\begin{array}{l}\text { ro } \\
0\end{array}$ & & & & $\stackrel{0}{\circ}$ & $\begin{array}{l}\infty \\
\hat{0}\end{array}$ & $\begin{array}{l}\infty \\
\infty \\
0 \\
0\end{array}$ & & & $\begin{array}{l}\tilde{O} \\
0 \\
0\end{array}$ & $\stackrel{B}{0}_{0}$ & \\
\hline J & $\begin{array}{l}\infty \\
\stackrel{i}{i}\end{array}$ & $\underset{\sim}{\stackrel{f}{*}}$ & $\begin{array}{l} \pm \\
\sigma\end{array}$ & $\begin{array}{l}\approx \\
\hat{0}\end{array}$ & ڤे & $\hat{a}$ & $\stackrel{ \pm}{-}$ & $\stackrel{\cong}{=}$ & $\stackrel{\Omega}{=}$ & $\underset{-1}{-}$ & ì & $\hat{\hat{i}}$ & $\stackrel{8}{i}$ & $\stackrel{0}{\mathfrak{r}}$ & $\underset{\mathcal{I}}{-}$ & $\vec{r}$ & $\begin{array}{l}\tilde{f} \\
i\end{array}$ & $\begin{array}{l}\tilde{\sigma} \\
\tilde{m}\end{array}$ & $\begin{array}{l}a \\
\infty \\
i\end{array}$ & $\stackrel{\bullet}{\bullet}$ & $\stackrel{i}{i}$ & $\stackrel{m}{0}$ & $\stackrel{8}{\stackrel{B}{-}}$ \\
\hline$\infty$ & $\begin{array}{l}\text { İ } \\
\text { ô }\end{array}$ & $\stackrel{1}{3}$ & $\frac{\Delta}{0}$ & $\stackrel{\infty}{0}$ & $\hat{0}$ & $\stackrel{2}{\circ}$ & $\frac{\infty}{0}$ & 今. & î & $\because$ & $\tilde{3}$ & fo & $\vec{m}$ & $\stackrel{\infty}{\stackrel{\infty}{0}}$ & $\stackrel{R}{0}$ & $\stackrel{\text { ?f }}{\circ}$ & $\begin{array}{c}0 \\
0 \\
0 \\
0\end{array}$ & 号. & iे & $\begin{array}{l}0 \\
\dot{m} \\
0\end{array}$ & \begin{tabular}{l}
0 \\
\multirow{2}{*}{} \\
0
\end{tabular} & 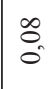 & तิ \\
\hline$a$ & $\begin{array}{l}\text { n. } \\
\stackrel{\sim}{n}\end{array}$ & $\begin{array}{l}\hat{\hat{o}} \\
\text { cे }\end{array}$ & $\begin{array}{l}\hat{2} \\
\stackrel{m}{m}\end{array}$ & $\begin{array}{l}\infty \\
\infty \\
\dot{m}\end{array}$ & $\begin{array}{c}m \\
\tilde{m} \\
\dot{m}\end{array}$ & $\begin{array}{l}0 \\
\text { m } \\
\text { m }\end{array}$ & $\begin{array}{l}\text { م } \\
\text { nn }\end{array}$ & $\begin{array}{l}\infty \\
\tilde{m} \\
\tilde{m}\end{array}$ & $\begin{array}{l}\text { J } \\
\text { @ }\end{array}$ & $\begin{array}{l}\infty \\
\infty \\
\infty \\
\text { nf }\end{array}$ & $\begin{array}{l}\tilde{\infty} \\
\infty \\
\tilde{i}^{0}\end{array}$ & $\underset{\stackrel{\sim}{\sim}}{\stackrel{ \pm}{\sim}}$ & $\begin{array}{l}m \\
\text { in } \\
\text { m }\end{array}$ & $\begin{array}{l}\text { ते } \\
\text { ले }\end{array}$ & $\begin{array}{l}8 \\
\text { i } \\
\text { r }\end{array}$ & $\begin{array}{l}8 \\
0 \\
\text { in }\end{array}$ & $\begin{array}{l}0 \\
0 \\
0 \\
0 \\
i\end{array}$ & 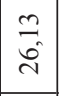 & $\begin{array}{l}0 \\
\text { N } \\
\text { n }\end{array}$ & $\begin{array}{l}\vec{n} \\
\vec{\lambda}\end{array}$ & 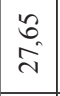 & $\stackrel{\vec{\sim}}{\stackrel{\vec{\lambda}}{2}}$ & $\begin{array}{l}\text { సे } \\
\text { fे }\end{array}$ \\
\hline$\vec{\omega}$ & $\stackrel{i}{\sim}$ & $\stackrel{\infty}{\stackrel{2}{r}}$ & $\begin{array}{l}\dot{m} \\
\stackrel{r}{+}\end{array}$ & $\stackrel{ }{m}$ & $\stackrel{g}{\rightarrow}$ & $\stackrel{+}{\infty}$ & $\begin{array}{l}\stackrel{\sim}{\sim} \\
\stackrel{\sim}{n}\end{array}$ & $\stackrel{\tilde{c}}{\sim}$ & $\stackrel{n}{n}$ & $\stackrel{n}{=}$ & $\begin{array}{l}\stackrel{\otimes}{\infty} \\
+\end{array}$ & $\begin{array}{l}\stackrel{\sim}{\sim} \\
\underset{\sim}{*}\end{array}$ & $\stackrel{\Delta}{\rightarrow}$ & $\vec{m}$ & $\stackrel{\circ}{=}$ & $\underset{r}{\stackrel{d}{r}}$ & $\stackrel{?}{\stackrel{f}{\sim}}$ & $\frac{0}{6}$ & $\stackrel{\text { qf }}{\text { i }}$ & $\begin{array}{l}0 \\
\stackrel{\sigma}{*}\end{array}$ & $\begin{array}{l}\infty \\
\infty \\
\infty \\
n\end{array}$ & $\stackrel{\Omega}{\curvearrowright}$ & $\stackrel{\cong}{=}$ \\
\hline « & $\begin{array}{l}\text { in } \\
\stackrel{\sim}{n}\end{array}$ & $\frac{n}{a}$ & $\begin{array}{l}\infty \\
\infty \\
\dot{J}\end{array}$ & $\frac{\vec{n}}{\vec{n}}$ & $\frac{\infty}{\overrightarrow{\mathrm{s}}}$ & $\begin{array}{l}8 \\
8 \\
\text { i }\end{array}$ & कु. & $\begin{array}{l}\tilde{b} \\
\text { s. }\end{array}$ & $\begin{array}{l}5 \\
\text { in }\end{array}$ & 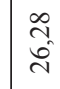 & 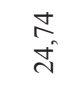 & 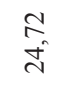 & $\hat{\imath}$ & $\begin{array}{l}\tilde{n} \\
\tilde{n}\end{array}$ & $\begin{array}{l}\hat{\text { in }} \\
\text { a }\end{array}$ & $\begin{array}{l}\stackrel{\vec{i}}{\hat{i}} \\
\text { }\end{array}$ & 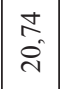 & $\begin{array}{l}\vec{F} \\
\vec{i} \\
\text { in }\end{array}$ & 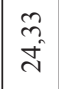 & $\begin{array}{l}\tilde{y} \\
\tilde{\lambda}\end{array}$ & $\begin{array}{l}\tilde{\alpha} \\
\hat{i}\end{array}$ & 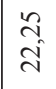 & $\begin{array}{l}8 \\
\text { ते }\end{array}$ \\
\hline 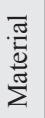 & 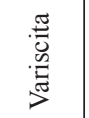 & 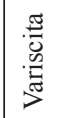 & 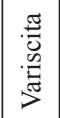 & 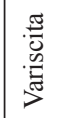 & 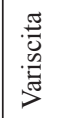 & 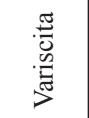 & 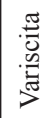 & 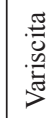 & 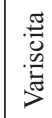 & 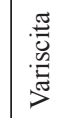 & 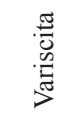 & 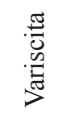 & 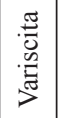 & 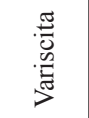 & 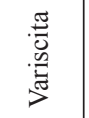 & 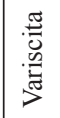 & 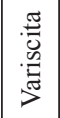 & 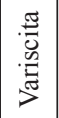 & 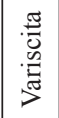 & 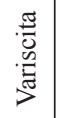 & 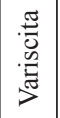 & 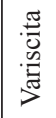 & 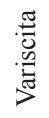 \\
\hline 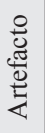 & 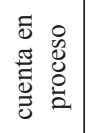 & 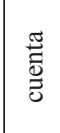 & 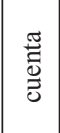 & 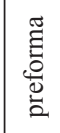 & 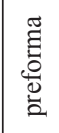 & 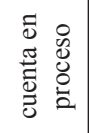 & 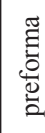 & 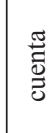 & 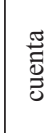 & 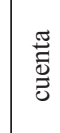 & 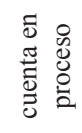 & 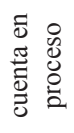 & 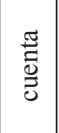 & 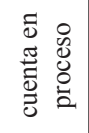 & 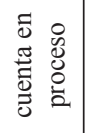 & 营 & 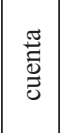 & 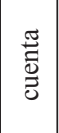 & 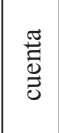 & 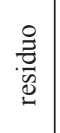 & 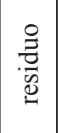 & 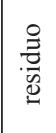 & $\frac{\widetilde{J}}{\frac{\tilde{g}}{\tilde{g}}}$ \\
\hline 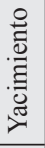 & 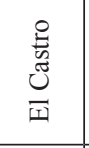 & 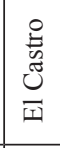 & 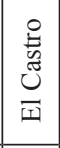 & $\begin{array}{l}\frac{8}{5} \\
\frac{7}{\tilde{J}} \\
\frac{1}{I}\end{array}$ & 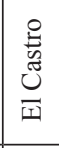 & 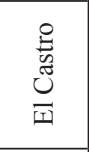 & 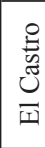 & 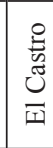 & 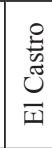 & 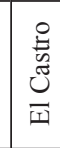 & 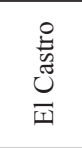 & $\begin{array}{l}\stackrel{8}{y_{0}} \\
\stackrel{\Xi}{\Psi}\end{array}$ & 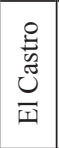 & 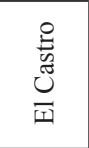 & 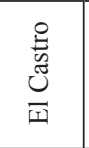 & 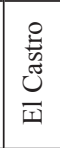 & 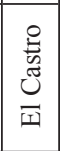 & 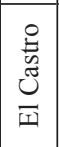 & 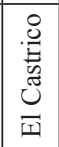 & 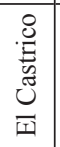 & 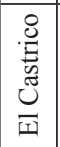 & 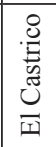 & 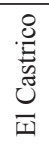 \\
\hline 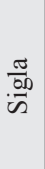 & $\begin{array}{l}\frac{\infty}{0} \\
\stackrel{0}{\infty} \\
\stackrel{\infty}{\infty}\end{array}$ & 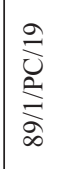 & 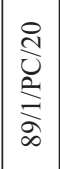 & 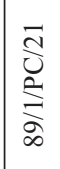 & 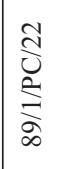 & $\begin{array}{l}\infty \\
0 \\
\stackrel{\infty}{2} \\
\stackrel{\infty}{2}\end{array}$ & $\stackrel{\infty}{\stackrel{\infty}{\circ}}$ & $\stackrel{\infty}{\stackrel{\infty}{\circ}}$ & $\stackrel{\infty}{\stackrel{\infty}{\circ}}$ & 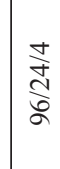 & 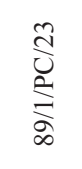 & 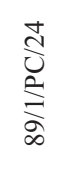 & 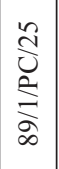 & 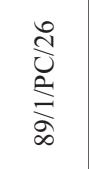 & $\begin{array}{l}0 \\
\stackrel{0}{*} \\
\stackrel{\infty}{*}\end{array}$ & $\begin{array}{l}\overrightarrow{0} \\
\stackrel{2}{2}\end{array}$ & \begin{tabular}{|c|}
$\tilde{D}$ \\
$\stackrel{1}{2}$ \\
$\stackrel{2}{\alpha}$ \\
$\infty$
\end{tabular} & 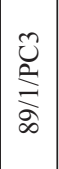 & 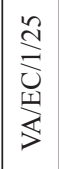 & 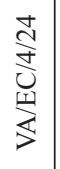 & 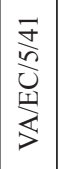 & 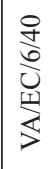 & 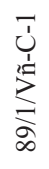 \\
\hline
\end{tabular}




\begin{tabular}{|c|c|c|c|c|c|c|c|c|c|c|c|c|c|c|c|c|c|}
\hline$\dot{N}$ & & $\begin{array}{l}\text { to } \\
\stackrel{0}{\circ}\end{array}$ & $\begin{array}{l} \pm \\
\stackrel{0}{0} \\
0\end{array}$ & $\overrightarrow{\sigma_{0}}$ & $\stackrel{8}{\circ}$ & $\stackrel{0}{0}$ & $\stackrel{\circ}{\circ}$ & $\tilde{\Xi}$ & $\begin{array}{l}\circ \\
\stackrel{0}{\circ} \\
\circ\end{array}$ & $\overline{8}$ & $\begin{array}{l}\infty \\
\stackrel{\infty}{\circ} \\
0\end{array}$ & $\stackrel{m}{0}$ & 范 & ठे. & $\begin{array}{l}\text { ते } \\
\text { ô. }\end{array}$ & $\begin{array}{l}m \\
0 \\
0\end{array}$ & $\begin{array}{l} \\
0 \\
0\end{array}$ \\
\hline$\ddot{\text { s }}$ & & & $\overrightarrow{\overrightarrow{0}_{0}}$ & $\vec{\partial}_{0}$ & $\begin{array}{l}0 \\
\stackrel{0}{0} \\
0\end{array}$ & & $\tilde{\Xi}_{0}$ & ठ̊. & 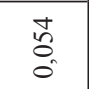 & $\begin{array}{l}\tilde{\delta} \\
\tilde{0} \\
0\end{array}$ & $\stackrel{\widetilde{\sigma}}{0}$ & : & 今̊ & $\begin{array}{l}0 \\
\stackrel{\infty}{0} \\
0 \\
0\end{array}$ & $\begin{array}{l}0 \\
\stackrel{0}{0}\end{array}$ & $\begin{array}{l}\overrightarrow{\vec{n}} \\
0.0\end{array}$ & 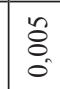 \\
\hline$\tilde{\psi}$ & 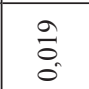 & $\begin{array}{l}0 \\
\stackrel{0}{0} \\
0\end{array}$ & & & & ô. & $\begin{array}{l}\vec{\delta} \\
\dot{0}\end{array}$ & \&. & $\begin{array}{l}\stackrel{2}{0} \\
0 \\
0\end{array}$ & $\begin{array}{l}\vec{J} \\
\dot{0} \\
0\end{array}$ & & & $\begin{array}{l}\tilde{O} \\
0 \\
0\end{array}$ & & $\begin{array}{l}\stackrel{\circ}{0} \\
\stackrel{0}{\circ}\end{array}$ & & $\begin{array}{l}\tilde{o}_{0} \\
\tilde{0}\end{array}$ \\
\hline ี & & $\stackrel{n}{\circ}$ & $\hat{8}$ & $\frac{n}{n}$ & $\begin{array}{l}\text { oे } \\
\text { o. }\end{array}$ & $\tilde{\circ}$ & $\stackrel{0}{0}$ & & $\begin{array}{l}\overrightarrow{0} \\
0_{0}\end{array}$ & ¿̊. & $\frac{J}{0}$ & $\begin{array}{l}\stackrel{0}{0} \\
\stackrel{0}{0}\end{array}$ & ôे & $\hat{s}_{0}$ & & $\begin{array}{l}\text { Or. } \\
\dot{0} \\
0\end{array}$ & \\
\hline z & & $\overrightarrow{0}$ & ${ }_{0}^{t}$ & $\stackrel{n}{q}$ & $\begin{array}{l}t \\
0 \\
0\end{array}$ & & & & $\stackrel{\delta}{0}$ & & $\hat{a}$ & $\stackrel{\circ}{=}$ & & $\stackrel{R}{0}$ & & $\stackrel{m}{\rightarrow}$ & \\
\hline 오 & $\tilde{2}$ & $\underset{\sim}{-}$ & fo & $\begin{array}{l}\infty \\
\infty \\
0\end{array}$ & $\overrightarrow{\tilde{\sigma}}$ & $\hat{\widehat{c}}$ & $\begin{array}{l}\stackrel{\infty}{\infty} \\
\text { ri }\end{array}$ & $\tilde{m}_{0}^{n}$ & $\begin{array}{l}\infty \\
\infty \\
\infty \\
0\end{array}$ & $\stackrel{n}{q}$ & 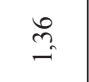 & $\begin{array}{l}\hat{\infty} \\
0_{0}\end{array}$ & $\overrightarrow{0}$ & $\begin{array}{l}\infty \\
\infty \\
0^{\circ}\end{array}$ & $\stackrel{\infty}{\stackrel{0}{0}}$ & $\begin{array}{l}\tilde{O} \\
0 \\
0\end{array}$ & \begin{tabular}{l}
$\infty$ \\
$\underset{\sim}{\infty}$ \\
\multirow{f}{*}{}
\end{tabular} \\
\hline ¿ & $\underset{m}{m}$ & $\underset{0}{J}$ & त̂ & & & ते & है & के & $\begin{array}{l}\stackrel{n}{3} \\
\text { on }\end{array}$ & ò & & & ते & & $\stackrel{+}{m_{0}}$ & & $\begin{array}{l}\text { స్ } \\
\text { of }\end{array}$ \\
\hline$>$ & & oे & & & & $\hat{0}$ & $\begin{array}{l}\infty \\
: \\
0\end{array}$ & & & $\hat{o}_{0}$ & & & & & & & \\
\hline$F$ & $\hat{\check{\sigma}}$ & $\stackrel{0}{0}$ & $\begin{array}{l}\overrightarrow{0} \\
0\end{array}$ & $\overrightarrow{\tilde{\sigma}}$ & $\stackrel{\Delta}{0}$ & $\begin{array}{l}\tilde{3} \\
0\end{array}$ & $\frac{\Delta}{0}$ & $\begin{array}{l}\infty \\
0 \\
0\end{array}$ & $\frac{2}{0}$ & $\stackrel{0}{\circ}$ & 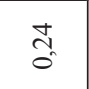 & $\frac{0}{0}$ & $\stackrel{7}{\circ}$ & $\frac{m}{0}$ & $\underset{0}{m}$ & $\begin{array}{l}\text { ñ } \\
\text { ñ. }\end{array}$ & ते \\
\hline$\tilde{J}$ & $\stackrel{3}{3}$ & $\stackrel{8}{8}$ & $\underset{\vec{\sigma}}{\partial}$ & $\underset{\sim}{\tilde{i}}$ & $\stackrel{\infty}{\stackrel{\sim}{\sim}}$ & $\stackrel{0}{\stackrel{0}{0}}$ & $\begin{array}{c}\infty \\
m_{0}^{\infty} \\
0\end{array}$ & $\vec{n}$ & $\begin{array}{l}\text { n. } \\
\text { ì }\end{array}$ & 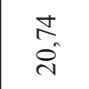 & $\begin{array}{l}\infty \\
\infty \\
\tilde{\lambda}\end{array}$ & $\begin{array}{l}\vec{\infty} \\
\stackrel{\infty}{0}\end{array}$ & $\frac{\ddot{n}}{m}$ & $\begin{array}{l}\hat{o} \\
i n\end{array}$ & ${ }_{-}^{a}$ & $\begin{array}{l}\infty \\
\infty \\
\vec{\sim}\end{array}$ & $\stackrel{+}{+}$ \\
\hline 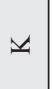 & $\begin{array}{l}\infty \\
\infty \\
0 \\
0\end{array}$ & $\stackrel{2}{\circ}$ & $\stackrel{m}{=}$ & $\stackrel{8}{:}$ & & & & $\stackrel{\infty}{\stackrel{0}{0}}$ & $\begin{array}{l}\stackrel{0}{0} \\
0\end{array}$ & ò & $\stackrel{8}{-}$ & $\hat{s}$ & $\begin{array}{l}n \\
n \\
0\end{array}$ & 3 & $\overrightarrow{\infty_{0}}$ & $\hat{\sigma}$ & $\begin{array}{l}\vec{\infty} \\
\sigma_{0}\end{array}$ \\
\hline $\bar{\tau}$ & $\stackrel{\widehat{N}}{-}$ & $\stackrel{m}{m}$ & $\frac{\partial}{m}$ & बे & $\begin{array}{l}n \\
0 \\
0\end{array}$ & $\stackrel{\sim}{\sim}$ & 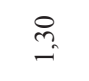 & $\begin{array}{l}\infty \\
\stackrel{\infty}{-}\end{array}$ & $\stackrel{ \pm}{=}$ & $\stackrel{+}{\rightarrow}$ & $\frac{\infty}{\vec{i}}$ & $\frac{\infty}{\vec{i}}$ & $\stackrel{8}{-}$ & $\stackrel{2}{=}$ & to & $\stackrel{\infty}{\rightarrow}$ & $\stackrel{8}{-}$ \\
\hline is & तु & 8 & $\stackrel{\overbrace{}}{-}$ & $\stackrel{\infty}{\infty}_{0}^{\infty}$ & $\stackrel{8}{0}$ & तु & 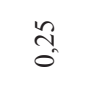 & $\begin{array}{l}0 \\
n_{0}^{2}\end{array}$ & $\begin{array}{l}\text { i } \\
\text { in }\end{array}$ & ते & $\hat{a}_{-}$ & $\stackrel{m}{\rightarrow}$ & $\stackrel{\cong}{\sim}$ & $\overrightarrow{\sigma_{i}}$ & $\hat{n}$ & $\hat{\vec{n}}$ & $=$ \\
\hline$a$ & $\begin{array}{l}\text { oे } \\
\text { iे }\end{array}$ & $\begin{array}{l}\text { ले } \\
\text { aे }\end{array}$ & $\begin{array}{l}\tilde{\alpha} \\
\hat{d}\end{array}$ & & & $\begin{array}{l}\text { } \\
\text { }\end{array}$ & 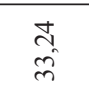 & $\underset{\stackrel{\sim}{m}}{\vec{m}}$ & $\frac{n}{a}$ & $\begin{array}{l}\stackrel{\text { I }}{=} \\
=\end{array}$ & & & $\begin{array}{l}\dot{\infty}_{\infty} \\
\infty\end{array}$ & $\stackrel{ \pm}{0}$ & $\begin{array}{l}\text { वे. } \\
\text { वे}\end{array}$ & \begin{tabular}{l}
$\infty$ \\
\multirow{0}{0}{} \\
0
\end{tabular} & $\begin{array}{l}0 \\
\sim \\
\tilde{d}\end{array}$ \\
\hline$\overline{\mathrm{s}}$ & $\begin{array}{l}0 \\
\text { fó } \\
6\end{array}$ & $\hat{\alpha}$ & $\stackrel{2}{\approx}$ & बे & $\begin{array}{l}d \\
0 \\
\infty \\
\infty\end{array}$ & $\underset{i}{\mathrm{i}}$ & $\stackrel{\vec{n}}{\rightarrow}$ & 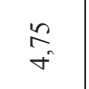 & $\begin{array}{l}\infty \\
\infty \\
\infty \\
0\end{array}$ & $\begin{array}{l}\vec{\circ} \\
\dot{f}\end{array}$ & $\begin{array}{l}\infty \\
\stackrel{0}{2}\end{array}$ & $\begin{array}{l}n \\
\tilde{n} \\
\ddot{n}\end{array}$ & $\underset{0}{8}$ & \begin{tabular}{l}
$\infty$ \\
\multirow{\Xi}{\pm}{} \\
\end{tabular} & $\begin{array}{c}\hat{n} \\
\stackrel{m}{\Rightarrow}\end{array}$ & $\begin{array}{l}\stackrel{i}{N} \\
\vec{i}\end{array}$ & $\begin{array}{l}q \\
\stackrel{9}{q}\end{array}$ \\
\hline « & $\underset{\tilde{i}}{\hat{i}}$ & 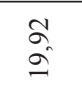 & $\stackrel{\circ}{\stackrel{1}{\circ}}$ & $\tilde{m}$ & $\begin{array}{l}\infty \\
i \\
i\end{array}$ & $\begin{array}{l}\overrightarrow{0} \\
\text { a } \\
\text { d }\end{array}$ & $\begin{array}{l}\vec{b} \\
\text { id }\end{array}$ & $\begin{array}{l}\overline{\text { ते }} \\
\text { in }\end{array}$ & $\hat{\sigma}$ & 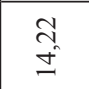 & $\stackrel{n}{\rightarrow}$ & $\underset{i}{i}$ & $\stackrel{\tilde{n}}{\stackrel{\Omega}{\Omega}}$ & $\underset{-}{ \pm}$ & $\begin{array}{l}\tilde{\vartheta} \\
\infty \\
\infty\end{array}$ & 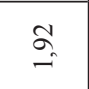 & $\begin{array}{l}\infty \\
\stackrel{\infty}{n} \\
=\end{array}$ \\
\hline 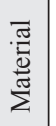 & 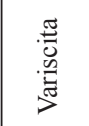 & 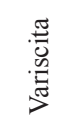 & 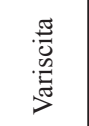 & 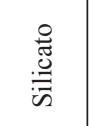 & 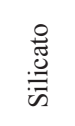 & 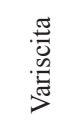 & 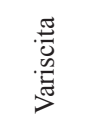 & 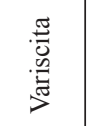 & 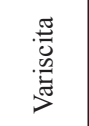 & 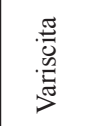 & 莺 & 莺 & 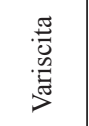 & 莺 & 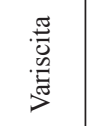 & 莺 & 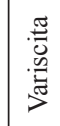 \\
\hline 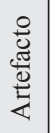 & $\begin{array}{l}\text { 芯 } \\
\text { एँ }\end{array}$ & $\begin{array}{l}\frac{\pi}{\tilde{J}} \\
\overline{\tilde{J}}\end{array}$ & 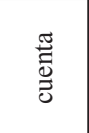 & $\begin{array}{l}\text { 芯 } \\
\text { एँ }\end{array}$ & $\begin{array}{l}\frac{\pi}{\tilde{\Xi}} \\
\frac{\tilde{g}}{0}\end{array}$ & 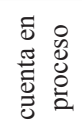 & $\begin{array}{l}\text { 芯 } \\
\text { 㫕 }\end{array}$ & 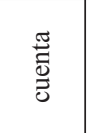 & 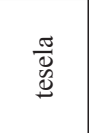 & 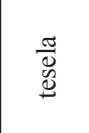 & 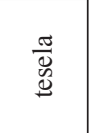 & $\begin{array}{l}\frac{\pi}{0} \\
0 \\
0\end{array}$ & $\begin{array}{l}\frac{\pi}{0} \\
\underline{W}\end{array}$ & $\begin{array}{l}\frac{\pi}{U !} \\
\underline{W}\end{array}$ & $\begin{array}{l}\stackrel{g}{\Xi} \\
\stackrel{0}{0} \\
\stackrel{0}{0}\end{array}$ & $\begin{array}{l}\frac{\pi}{0} \\
\frac{0}{0}\end{array}$ & $\begin{array}{l}\stackrel{\circ}{\bar{z}} \\
. \\
ٌ\end{array}$ \\
\hline 菢 & 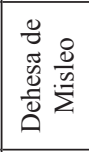 & 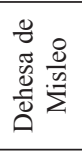 & 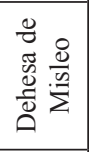 & 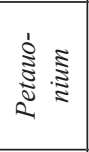 & 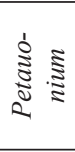 & 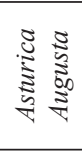 & 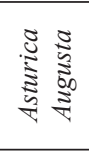 & 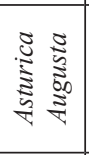 & 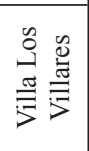 & 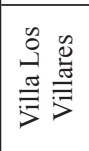 & 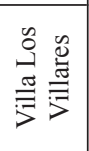 & 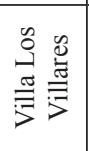 & 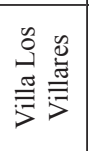 & 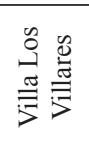 & 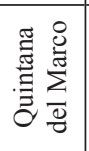 & 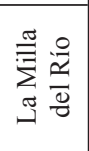 & 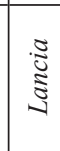 \\
\hline 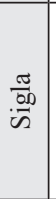 & $\underset{\infty}{\vec{E}}$ & $\sum_{\infty}^{\infty}$ & $\underset{\infty}{\stackrel{S}{E}}$ & $\begin{array}{l}\stackrel{m}{\Rightarrow} \\
\stackrel{m}{n} \\
\stackrel{2}{a}\end{array}$ & 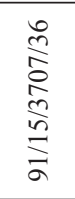 & 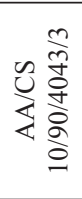 & 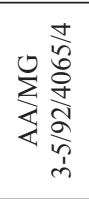 & 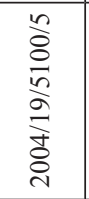 & $\overrightarrow{\widetilde{n}}$ & 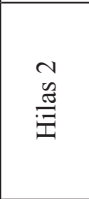 & 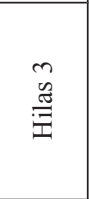 & 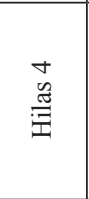 & 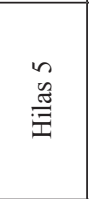 & $\begin{array}{l}0 \\
\stackrel{0}{0} \\
\stackrel{\Xi}{\Xi}\end{array}$ & 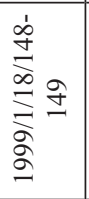 & 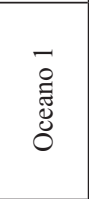 & $\begin{array}{l}\overline{5} \\
\stackrel{\alpha}{\sigma}\end{array}$ \\
\hline
\end{tabular}




\begin{tabular}{|c|c|c|c|c|c|c|c|c|c|c|c|c|c|c|c|c|c|c|}
\hline$\dot{N}$ & \%̊. & $\begin{array}{l}\tilde{\delta} \\
\delta \\
0\end{array}$ & $\tilde{\delta}_{0}$ & \& & $\stackrel{\circ}{\circ}$ & $\stackrel{m}{0}$ & ¿े & ò & $\stackrel{\circ}{\circ}$ & ¿̊. & $\stackrel{m}{0}$ & $\frac{\partial}{0}$ & $\overrightarrow{0}$ & $\stackrel{8}{\circ}$ & $\stackrel{0}{0}_{0}^{\infty}$ & $\begin{array}{l}\stackrel{0}{0} \\
0 \\
0\end{array}$ & oे & $\hat{\overline{0}}$ \\
\hline$\ddot{\omega}$ & 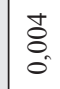 & 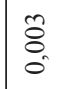 & 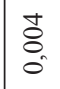 & $\begin{array}{l}\overrightarrow{0} \\
\dot{0}\end{array}$ & तె & $\begin{array}{l}\text { 我 } \\
0 \\
0\end{array}$ & $\begin{array}{l}0 \\
0 \\
0 \\
0\end{array}$ & $\begin{array}{l}\text { ô. } \\
\text { Oे }\end{array}$ & $\begin{array}{l}\infty \\
\stackrel{\infty}{0} \\
0 \\
0\end{array}$ & $\begin{array}{l}\infty \\
0 \\
0 \\
0\end{array}$ & $\begin{array}{l}\tilde{O} \\
\dot{0}\end{array}$ & \begin{tabular}{l}
$\infty$ \\
\multirow{0}{0}{} \\
0
\end{tabular} & $\stackrel{8}{0}$ & $\stackrel{0}{0}$ & $\begin{array}{l}\vec{\circ} \\
\dot{0}\end{array}$ & 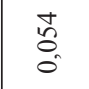 & $\begin{array}{l}\text { Iै } \\
0 \\
0\end{array}$ & $\begin{array}{l} \pm \\
0 \\
0\end{array}$ \\
\hline \& & $\begin{array}{l}\text { Ho } \\
\text { o. }\end{array}$ & $\begin{array}{l}1 \\
0 \\
0 \\
0 \\
0\end{array}$ & n̂. & $\frac{a}{\square}$ & $\stackrel{8}{0}$ & & & & & & & $\begin{array}{l}\tilde{o} \\
\tilde{0} \\
0\end{array}$ & & & & & & \\
\hline ี & & & & & & & ठ̊ & \& & & $\begin{array}{l}\infty \\
\stackrel{0}{0} \\
0\end{array}$ & & $\overrightarrow{0}$ & & $\begin{array}{l}0 \\
\text { O্. } \\
0\end{array}$ & $\stackrel{\infty}{\circ}$ & $\begin{array}{l}\vec{\delta} \\
\dot{0}\end{array}$ & $\stackrel{0}{0}$ & $\begin{array}{l}\infty \\
\stackrel{0}{0} \\
0\end{array}$ \\
\hline z & & & & & & & & $\stackrel{n}{\circ}$ & $\vec{s}$ & $:$ & $\tilde{0}$ & & S. & $\tilde{3}$ & $\stackrel{o}{o}$ & $\stackrel{+}{\stackrel{0}{0}}$ & $\stackrel{\circ}{-}$ & $\stackrel{t}{0}$ \\
\hline ix & $\underset{\substack{0 \\
\dot{r}}}{4}$ & $\begin{array}{l}\tilde{\infty} \\
i \\
c\end{array}$ & $\underset{f}{f}$ & $\hat{\mathrm{m}}$ & $\underset{f}{\text { f }}$ & $\stackrel{\text { gे }}{\rightarrow}$ & $\begin{array}{l}\infty \\
\infty \\
0\end{array}$ & $\stackrel{N}{0}$ & $\stackrel{8}{\circ}$ & $\cong$ & $\hat{n}$ & in & $\stackrel{\mathbb{J}}{=}$ & $\begin{array}{l}\infty \\
\infty \\
0\end{array}$ & $\stackrel{\infty}{\circ}$ & $\stackrel{f}{-}$ & $\stackrel{q}{q}$ & $\stackrel{\overbrace{}}{\sim}$ \\
\hline む & : & in & $\begin{array}{c}\hat{y} \\
\hat{0}\end{array}$ & $\begin{array}{l}0 \\
0 \\
0 \\
0\end{array}$ & $\tilde{n}_{0}^{n}$ & $\begin{array}{l}\vec{n} \\
0\end{array}$ & ते & & & & & : & & & & & & \\
\hline$>$ & $\begin{array}{l}5 \\
0 \\
0\end{array}$ & 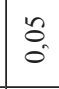 & & $\stackrel{\infty}{:}$ & $\hat{0}$ & & & & & & & & & & & & & \\
\hline $\mathrm{F}$ & $\vec{c}$ & \begin{tabular}{l}
$\infty$ \\
\multirow{0}{0}{} \\
$\tilde{0}$
\end{tabular} & ते & $\frac{\Delta}{0}$ & $\stackrel{7}{0}$ & $\begin{array}{l}\text { İ } \\
\text { ó }\end{array}$ & $\begin{array}{l}0 \\
\stackrel{0}{0}\end{array}$ & $\stackrel{\circ}{\circ}$ & $\frac{\Delta}{0}$ & $\stackrel{\infty}{0}$ & $\begin{array}{l}\text { त̂ } \\
\text { ô }\end{array}$ & $\stackrel{m}{0}$ & $\stackrel{7}{0}$ & $\overrightarrow{\tilde{o}}$ & $\frac{\pi}{0}$ & त̃ & $\stackrel{n}{0}$ & के \\
\hline$\tilde{U}$ & $\frac{t}{0}$ & $\stackrel{\infty}{\circ}$ & $\stackrel{\sigma}{-}$ & $\begin{array}{l}\stackrel{\circ}{\sim} \\
\sim\end{array}$ & $\vec{n}$ & $\stackrel{n}{\sim}$ & à & $\overrightarrow{D_{0}}$ & 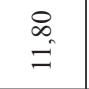 & $\begin{array}{l}0 \\
\text { م. } \\
0\end{array}$ & $\begin{array}{l}\bar{\Xi} \\
\stackrel{\Delta}{-}\end{array}$ & $\begin{array}{l}\vec{a} \\
\stackrel{0}{-}\end{array}$ & $\begin{array}{l}\infty \\
\vec{N}\end{array}$ & $\begin{array}{l}\stackrel{8}{0} \\
\text { i }\end{array}$ & $\begin{array}{l}8 \\
\text { - }\end{array}$ & $\stackrel{n}{\stackrel{n}{=}}$ & $\stackrel{\tilde{n}}{=}$ & $\begin{array}{l}\stackrel{m}{\stackrel{\infty}{\infty}} \\
\stackrel{\infty}{0}\end{array}$ \\
\hline$\searrow$ & $\begin{array}{l}0 \\
0 \\
0\end{array}$ & $\begin{array}{l}\infty \\
n \\
0 \\
0\end{array}$ & $\begin{array}{l}n \\
\infty \\
0 \\
0\end{array}$ & $\begin{array}{l}\tilde{b} \\
i\end{array}$ & $\stackrel{g}{\rightarrow}$ & 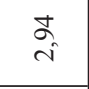 & $\underset{\text { i }}{\text { i }}$ & $\begin{array}{r}\text { g. } \\
\text { on }\end{array}$ & $\exists$ & $\stackrel{\hat{\sigma}}{-}$ & $\stackrel{0}{:}$ & $\stackrel{0}{0}$ & $\hat{o}$ & $\stackrel{\infty}{\circ}$ & $\begin{array}{l}\infty \\
\infty \\
0\end{array}$ & $\stackrel{\overbrace{}}{\leftrightarrows}$ & $\vec{\sigma}_{-}$ & $\stackrel{\text { مे }}{\rightarrow}$ \\
\hline $\bar{U}$ & $\stackrel{\infty}{\rightarrow}$ & $\stackrel{n}{=}$ & $\stackrel{\infty}{\stackrel{\sim}{\sim}}$ & $\underset{-}{\mathbb{G}}$ & $\begin{array}{l}\infty \\
\infty \\
0\end{array}$ & ते & : & $\stackrel{\cong}{=}$ & $\stackrel{f}{-}$ & $\stackrel{\leftrightarrow}{\rightarrow}$ & $\vec{n}$ & $\begin{array}{l}\infty \\
n \\
0\end{array}$ & $\vec{m}$ & $\Xi$ & $\stackrel{f}{-}$ & $\stackrel{\infty}{\stackrel{\infty}{\rightarrow}}$ & $\stackrel{\vec{m}}{\rightarrow}$ & $\begin{array}{l}\vec{\infty} \\
0 \\
0\end{array}$ \\
\hline is & $\frac{0}{0}$ & $\stackrel{n}{0}$ & $\begin{array}{l}0 \\
\text { In } \\
0\end{array}$ & $\stackrel{\partial}{\circ}$ & $\stackrel{g}{o}$ & $\begin{array}{l}0 \\
\tilde{r}_{0}\end{array}$ & $\stackrel{\infty}{0}_{0}^{\infty}$ & $\underset{-}{\mathbb{G}}$ & $\begin{array}{l}\hat{0} \\
i\end{array}$ & $\begin{array}{l}\hat{8} \\
+\end{array}$ & $\tilde{\alpha}$ & 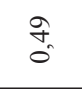 & $\begin{array}{l}\infty \\
m \\
n\end{array}$ & $\begin{array}{l}\text { nn } \\
\text { in }\end{array}$ & $\vec{\sigma}$ & $\begin{array}{l}50 \\
\text { in }\end{array}$ & $\stackrel{\circ}{\stackrel{m}{n}}$ & $\underset{r}{\stackrel{d}{r}}$ \\
\hline$a$ & $\begin{array}{l}\infty \\
\hat{\tilde{m}} \\
\end{array}$ & 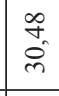 & $\stackrel{m}{\vec{i}}$ & $\begin{array}{l}\stackrel{7}{\simeq} \\
=\end{array}$ & 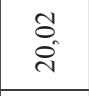 & $\begin{array}{l}\text { ? } \\
\stackrel{0}{\longrightarrow}\end{array}$ & $\stackrel{ \pm}{=}$ & & $=$ & & & & & & & & & $\frac{n}{0}$ \\
\hline$\vec{c}$ & $\begin{array}{l}8 \\
+ \\
\end{array}$ & 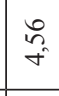 & $\begin{array}{l}\mathcal{S} \\
\mathcal{I} \\
\end{array}$ & $\hat{\imath}$ & $\stackrel{?}{=}$ & $\begin{array}{l}\text { 品 } \\
\text { J } \\
\end{array}$ & $\stackrel{2}{\stackrel{\Xi}{=}}$ & $\begin{array}{l} \pm \\
\infty \\
\text { ते }\end{array}$ & $\begin{array}{l}\circ \\
\text { ni }\end{array}$ & $\begin{array}{l}0 \\
\text { in }\end{array}$ & $\begin{array}{l}\text { i } \\
\stackrel{\text { ஸे }}{ }\end{array}$ & $\overrightarrow{\vec{n}}$ & $\stackrel{7}{2}$ & $\begin{array}{l}\overrightarrow{0} \\
\stackrel{0}{0}\end{array}$ & 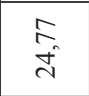 & $\begin{array}{l}\infty \\
\stackrel{\leftrightarrow}{0}\end{array}$ & $\begin{array}{l}n \\
\hat{s} \\
i\end{array}$ & $\stackrel{\infty}{\stackrel{\infty}{=}}$ \\
\hline « & $\begin{array}{l}\overrightarrow{0} \\
\text { î }\end{array}$ & $\begin{array}{l}\infty \\
\vec{i}\end{array}$ & $\stackrel{\infty}{\stackrel{0}{0}}$ & $\begin{array}{l}\stackrel{0}{2} \\
\stackrel{2}{2}\end{array}$ & $\begin{array}{l}\stackrel{2}{2} \\
\stackrel{2}{2}\end{array}$ & $\begin{array}{l}\infty \\
m \\
m\end{array}$ & $\begin{array}{l}\stackrel{\infty}{\infty} \\
=\end{array}$ & $\begin{array}{l}\dot{+} \\
\stackrel{+}{*}\end{array}$ & $\vec{i}$ & $\underset{n}{\mathbb{N}}$ & $\underset{i}{8}$ & $\underset{r}{\stackrel{g}{r}}$ & $\stackrel{\approx}{\tilde{i}}$ & 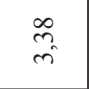 & $\vec{m}$ & $\begin{array}{l}\infty \\
\tilde{r}_{0}\end{array}$ & $\stackrel{n}{n}$ & $\begin{array}{l}8 \\
\text { in }\end{array}$ \\
\hline 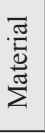 & 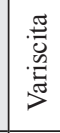 & 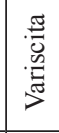 & 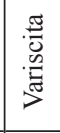 & 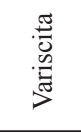 & 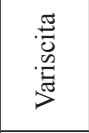 & 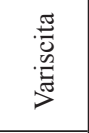 & 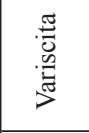 & & & & & & & & & & & \\
\hline 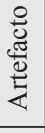 & $\stackrel{\circ}{\stackrel{\Xi}{\Xi}}$ & $\frac{\circ}{\stackrel{\bar{z}}{o g}}$ & 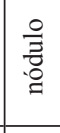 & $\begin{array}{l}\frac{\pi}{0} \\
\frac{0}{0}\end{array}$ & $\begin{array}{l}\frac{\pi}{0} \\
0 \\
0 \\
d\end{array}$ & $\begin{array}{l}\frac{\pi}{0} \\
\mathbb{J} \\
0\end{array}$ & $\begin{array}{l}\frac{\pi}{0} \\
0 \\
0\end{array}$ & $\begin{array}{l}\frac{\pi}{0} \\
0 \\
0\end{array}$ & $\begin{array}{l}\frac{\pi}{0} \\
0 \\
0\end{array}$ & $\begin{array}{l}\frac{\pi}{0} \\
0 \\
0 \\
0\end{array}$ & $\begin{array}{l}\frac{\pi}{0} \\
0 \\
0\end{array}$ & $\begin{array}{l}\frac{\pi}{0} \\
0 \\
0 \\
0\end{array}$ & $\begin{array}{l}\frac{\pi}{0} \\
\stackrel{0}{0}\end{array}$ & $\begin{array}{l}\frac{\pi}{d} \\
0 \\
0\end{array}$ & $\begin{array}{l}\frac{\pi}{0} \\
\mathbb{0} \\
0\end{array}$ & 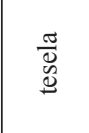 & $\begin{array}{l}\frac{\pi}{0} \\
\underline{J} \\
\underline{W}\end{array}$ & $\begin{array}{l}\text { 焉 } \\
\underline{\underline{S}}\end{array}$ \\
\hline 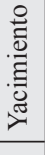 & 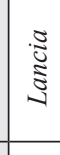 & 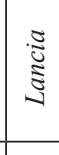 & 胥 & 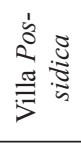 & 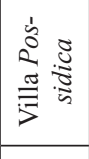 & 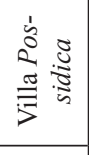 & 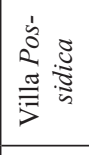 & 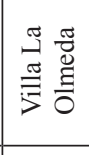 & 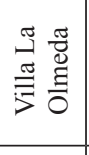 & 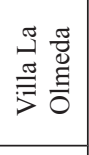 & 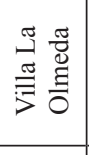 & 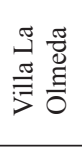 & 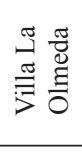 & 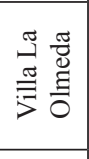 & 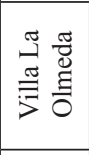 & 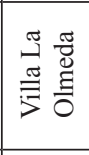 & 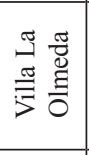 & 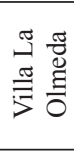 \\
\hline 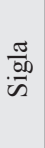 & $\begin{array}{l}\bar{\infty} \\
\stackrel{\infty}{\sigma}\end{array}$ & $\begin{array}{l}\bar{\alpha} \\
\stackrel{\alpha}{\sigma} \\
\stackrel{2}{2}\end{array}$ & $\begin{array}{l}\vec{\infty} \\
\stackrel{\sigma}{\sigma}\end{array}$ & $\begin{array}{l}\overrightarrow{0} \\
\bar{\Xi} \\
0 \\
0\end{array}$ & 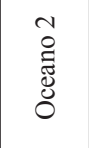 & $\begin{array}{l}n \\
\text { हूँ } \\
\tilde{\Xi} \\
0\end{array}$ & 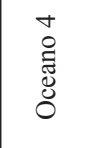 & $\begin{array}{l}\overline{3} \\
\bar{\Xi} \\
0\end{array}$ & $\begin{array}{l}n \\
\tilde{\Xi} \\
\tilde{\Xi} \\
0\end{array}$ & $\begin{array}{l}n \\
\tilde{\Xi} \\
\tilde{\Xi}\end{array}$ & 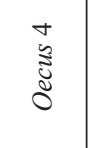 & $\begin{array}{l}n \\
\tilde{z} \\
\tilde{\Xi} \\
0\end{array}$ & $\begin{array}{l}0 \\
\vdots \\
\tilde{\Xi} \\
0\end{array}$ & $\begin{array}{l}\bar{y} \\
\tilde{\Xi} \\
0\end{array}$ & 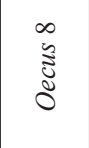 & $\begin{array}{l}\hat{\Xi} \\
\tilde{\Xi} \\
\delta\end{array}$ & $\begin{array}{l}\frac{0}{5} \\
\bar{z} \\
0\end{array}$ & $\begin{array}{l}\bar{\Xi} \\
\bar{\Xi} \\
0 \\
0\end{array}$ \\
\hline
\end{tabular}




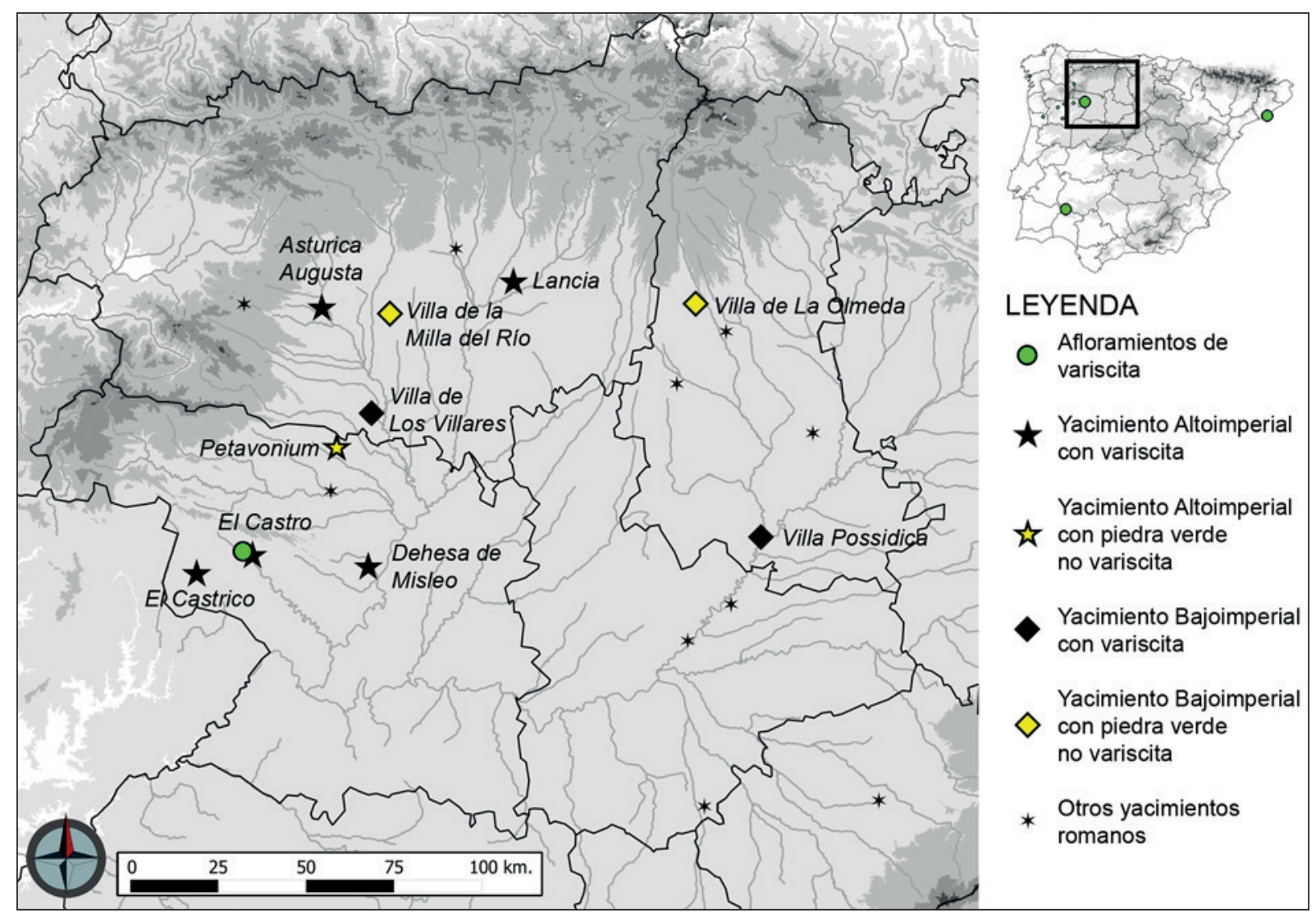

Figura 4. Localización de los diversos yacimientos tratados en el texto y de las minas de variscita peninsulares.

Por otro lado, el oficio de los musivarios se atestigua por un relieve, donde se ven diferentes artesanos en el momento en que cortan las teselas y se preparan para el transporte (Antiquarium, Ostia). Igualmente, queda manifiesto en el denominado Edicto de Precios (Edictum de Pretiis) del 301 d.C., norma promulgada por el emperador Diocleciano que fijaba los precios máximos de más de 1300 productos y establecía el coste de la mano de obra para producirlos: un tessellarius cobraba al día 50 denarios y 60 cobraría un mussiuarius, ambos sueldos inferiores al del pictor imaginarius, es decir, el pintor que diseñaba las imágenes. Por último, se tiene conocimiento del nombre de catorce artesanos por medio de las "firmas" presentes en los mosaicos.

La elaboración de los suelos musivarios era un trabajo en equipo, formado por hombres libres, libertos y esclavos, y donde cada uno tendría bien delimitadas sus funciones. Estos obradores tendrían un carácter itinerante y trabajarían, normalmente, en ámbitos regionales donde se aprovisionarían del material necesario. De la villa palentina de La Olmeda se conoce bien la procedencia de la mayoría de las teselas, de un lugar de la montaña cantábrica, entre Alar del Rey y Aguilar de Campoo (Regueras 2013: 79), lejano, por tanto de las minas zamoranas de variscita.

El trabajo de desbastado y la manufactura de las teselas se efectuarían en el propio lugar donde se iba a realizar el mosaico. Nuevamente, en La Olmeda se localizaron, en las habitaciones V-21 y V-35, teselas preparadas y placas de vidrio para futuras reparaciones, por lo que se tiende a identificar a ambas habitaciones como talleres (Abásolo y Martínez 2012: 50 y 60). Al igual que en la villa romana de Los Moros (La Serna, Palencia), donde se descubrió una habitación semisubterrénea con un depósito de materiales de un mosaísta y una gran acumulación de teselas amortizadas de un pavimento anterior que pudo fecharse a fines del siglo III (Nozal et al. 1995: 369-370 y 372; Regueras 2012: 79).

Por último, resulta curioso que en villas relativamente cercanas, y que presentan ambas mosaicos de gran calidad, como son La Olmeda y Possidica, en una no aparezcan teselas de variscita y en la otra sí. Esto puede tener una explicación bastante sencilla, los maestros musivarios trabajarían con los "productos naturales" 
que les ofrecía el entorno más inmediato (ya que transportar toneladas de "piedras" para la realización de mosaicos sería ridículo) y, que aparezcan teselas de variscita, dependería del musivario contratado para la obra. Al no tener un "taller fijo", los musivarios se irían desplazando de una villa a otra ofreciendo sus servicios y, seguramente, se llevarían con ellos planchas de diferentes colores (como las que aparecen en Los Villares) ya preparadas para la realización de teselas y fáciles de manejar y transportar. Piedras más difíciles de conseguir, como el caso de la variscita, también serían transportadas de un sitio a otro, pero solo por aquellos que conocieran su existencia. Siguiendo con este razonamiento, no todos los musivarios, sobre todo si no eran procedentes de la zona de Zamora, tendrían conocimiento de la existencia de la variscita $y$, por tanto, la explotación de las minas se realizaría de una forma más reducida.

\section{Agradecimientos}

Aprovechamos para dar las gracias a la Diputación de Palencia por permitirnos realizar los análisis in situ sobre el mosaico de Aquiles en Skyros de La Olmeda. Agradecimiento extensible al personal de la villa por las facilidades dadas a la hora de realizar nuestro trabajo. Igualmente, agradecemos la colaboración del personal del Museo Arqueológico de Palencia, del Museo Arqueológico de Zamora y del Museo Arqueológico de León por su ayuda desinteresada para la realización de este trabajo

\section{BIBLIOGRAFÍA}

Abásolo Álvarez, J.A. y Martínez, R. (2012): Villa romana La Olmeda. Guía arqueológica. Palencia, Diputación de Palencia.

Alonso, M.; Edo i Benaiges, M.; Gordo, L.; Millán, M., y Villalba, M. J. (1978): “Explotación minera neolítica en Can Tintoré (Gavà, Barcelona)". Pyrenae 13-14: 7-14.

Arribas, A.; Galán, E.; Martín-Pozas, J. M.; Nicolau, J. y Salvador, P. (1971): "Estudio mineralógico de la variscita de Palazuelo de las Cuevas, Zamora (España)". Studia Geologica Salmanticensia 2: 115-132.

Balagny, C. (1939): "Le mystere de la callais". Societé Archéologique de Nantes 79: 173-216.

Barstow, R. W. (1982): "Variscite from Hensbarrow china clay works, St. Austell, Cornwall". Mineralogical Magazine 46: 512.
Beckhoff, B.; Kanngiesse, B.; Langhoff, N.; Wedell, R. y Wolff, H. (2006): Handbook of practical X-ray fluorescence analysis. Springer.

Bordas, A.; Molinas, R.; Saa, M.; Melgarejo, J.-C. y Lehbib, S. (2009): "Les mines històriques al sector de la serra de les Ferreres: darreres aportacions a l'estudi de la Mineria del ferro a Gavà", en J. Bosch y F. Borrell (eds.), Intervencions arqueològiques a les Mines de Gavà (sector serra de les Ferreres). Anys 1998-2009: 247-262. Gavà (Barcelona), Museu de Gavà.

Bosch, J. y Borrell, F. (eds.) (2009): Intervencions arqueològiques a les Mines de Gavà (sector serra de les Ferreres). Anys 1998-2009. De la variscita al ferro: neolític $i$ antiquitat. Gavà (Barcelona), Museu de Gavà.

Burón Álvarez, M. (1997): El trazado urbano en las proximidades del Foro en Asturica Augusta. Memorias. Arqueología en Castilla y León 2. Salamanca, Junta de Castilla y León.

Carretero Vaquero, S. (1991): "Petavonium: historia de dos campamentos romanos". Revista de Arqueología 125: 30-39.

Carretero Vaquero, S. (2009): "Petavonium", el hogar hispano de la legión X "Gemina" y del ala II "Flavia”. Anuario del Instituto de Estudios Zamoranos Florián Ocampo 26: 13-44.

Criss, J.W. y Birks, L.S. (1968): “Calculation methods for fluorescent x-ray spectrometry. Empirical coefficients versus fundamental parameters". Analytical Chemistry 40: 1080-1086.

Domínguez-Bella, S. (2004): "Variscite, a prestige mineral in the Neolithic-Aeneolithic Europe. Raw material sources and possible distribution routes". Slovak Geological Magazine 10 (1-2): 147-152.

Edo i Benaiges, M.; Villalba, M. J. y Blasco, A. (1995): "La Calaíta en la Península Ibérica", en V. O. Jorge (ed.), $1^{\circ}$ Congresso de Arqueologia Peninsular. Actas VI: 127-168. Porto, Sociedade Portuguesa de Antropologia e Etnologia.

Edo i Benaiges, M.; Blasco, A.; Villalba, M. J.; Gimeno, D.; Fernández Turiel, J. L. y Plana, F. (1998): "La caracterización de la variscita del complejo minero de Can Tintorer. Una experiencia aplicada al conocimiento del sistema de bienes de prestigio durante el Neolítico", en J. Bernabeu, T. Orozco, y X. Terradas (eds.), Los recursos abióticos en la Prehistoria. Caracterización, aprovisionamiento e intercambio: 83-109. Valencia, Universitat de València.

Elam, W.; Shen, R.; Scruggs, B. y Nicolosi, J. (2004): "Accuracy of standardless FP analysis of bulk and 
thin film samples using a new atomic database". Advances in X-ray Analysis 47: 104-109.

Elton, N. J. (1996): "Variscite and Metavariscite from Gunheath China Clay Pit, St Austell, Cornwall". Mineralogical Magazine 60: 671-672.

Fernández González, J.J. (2012): “En torno a Villa Possidica y sus mosaicos: los trabajos de protección de 1991”, en C. Fernández y R. Bohigas (Coords.), In Durii regione romanitas. Estudios sobre la presencia romana en el valle del Duero en homenaje a Javier Cortes Álvarez de Miranda: 321-327. Palencia/ Santander.

Forestier, F. H.; Lasnier, B. y L’Helgouach, J. (1973a): "Découverte de minyulite en échantillons spectaculaires, de wavellite et de variscite dans les phtanites siluriens près de Pannecé (Loire- Atlantique)". $\mathrm{Bu}$ lletin de la Société Minéralogique de Cristallographie 96: 67-71.

Forestier, F. H.; Lasnier, B. y L'Helgouach, J. (1973b): “À propos de la "callaïs", découverte d'un gisement de variscite à Pannecé (Loire-Atlantique), analyse de quelques "perles vertes" néolithiques". Bulletin de la Société Préhistorique Française 70: 173-180.

Frantz, T.; Abramitis, D. H.; Borsch, L. y Wypyski, M. T. (2009): "Roman Variscite Beads: In Situ Analysis by X-ray Microdiffraction". The Metropolitan Museum of Art Bulletin 67(1): 20-25.

Garcia-Guinea, J.; Sapalski, C.; Cardenes, V. y Lombardero, M. (2000): "Mineral inlays in natural stone slabs: techniques, materials and preservation". Construction and Building Materials 14(6-7): $365-$ 373. http://hdl.handle.net/10261/67090.

Herbaut, F. y Querré, G. (2004): "La parure néolithique en variscite dans le sud de l'Armorique". Bulletin de la Société Préhistorique Française 101(3): 497-520.

Hutchinson, M. E. (1996): Gemmological work in the Ancient Monuments Laboratory 1980-1995. Londres, Historic Buildings and Monuments Commission for England.

Lheur, C. (1993): “Les minéralisations de l'ancienne carrière de La Floquerie près de Pannecé (Loire-Atlantique)". Le Cahier des Micromonteurs 4: 14-21.

Marcos Fierro, R. M. (1994): "La sustancia: naturaleza. Análisis petrográfico", en El mosaico de «Hilas y las ninfas». Museo de León: 65-75. Valladolid, Junta de Castilla y León.

Marini, C.; Gimeno, D. y Sistu, G. (1989): “Le mineralizzazioni a variscite del Sarrabus". Bolletino della Società Geologica Italiana 108: 357-367.
Martins Valls, R. y Delibes de Castro, G. (1979): "Hallazgos arqueológicos en la provincia de Zamora (VI)". BSAA XLV: 128-135.

Massé, R. (1971): "Découvert de minyulite, wavellite et variscite dans les phtanites de Pannecé". Bulletin de la Société Sciences Naturelles Ouest de la France LXIX: 12-15.

Meireles, C.; Ferreira, N. y Reis, M. L. (1987): "Variscite Occurrence in Silurian Formations from Northern Portugal". Comunicações Dos Serviços Geológicos de Portugal 73(1/2): 21-27.

Middleton, A.; La Niece, S.; Ambers, J.; Hook, D.; Hobbs, R. y Seddon, G. (2007): "An elusive stone: the use of variscite as a semi-precious stone". The British Museum Technical Research Bulletin 1: 29-34.

Moro Benito, M. C.; Cembranos Pérez, M. L. y Fernández-Fernández, A. (1995): “Estudio mineralógico de las variscitas y turquesas silúricas de Punta Corveiro (Pontevedra, España)". Geogaceta 18: 176-179.

Nocete, F. y Linares, J. A. (1999): "Las primeras sociedades mineras en Huelva", en Historia de la provincia de Huelva: 49-64. Huelva, Mediterráneo .

Nozal, M.; Puertas, F. y Ríos, D. (1995): “La Villa romana de "Los Moros", La Serna (Palencia). Trabajos de prospección y sondeo", en M.V. Calleja González (coord.), Actas del III Congreso de Historia de Palencia: 365-380. Palencia (1995), Palencia, Diputación Provincial de Palencia.

Odriozola, C. P.; Linares Catela, J. A. y Hurtado Pérez, V. (2010): "Variscite source and source analysis: testing assumptions at Pico Centeno (Encinasola, Spain)". Journal of Archaeological Science 37(12): 3146-3157. doi:10.1016/j.jas.2010.07.016.

Palol, P. de (1963): "El mosaico de tema oceánico de la villa de Dueñas (Palencia)". BSAA XXIX: 5-35.

Palol, P. de y Cortes, J. (1974): La villa romana de La Olmeda, Pedrosa de la Vega (Palencia). Acta Arqueológica Hispánica 7. Madrid.

Pérez Macías, J. A. (2010): "Un asentamiento del III Milenio A.C. en La Lapa (Encinasola, Huelva)", en XXII Jornadas de la Comarca de la Sierra: 269285. Huelva, Diputación de Huelva.

Regueras, F.; Yagüe, P.L. y Marcos, R. (1994): El mosaico de "Hilas y las ninfas", Museo de León: rapto y rescate del héroe. León, Junta de Castilla y León.

Regueras Grande, F. (2013): Villas romana del Duero. Historia de un paisaje olvidado. Valladolid, Asociación Domvs Pvcelae.

Revilla, R.; Palol, P. de y Cuadros, A. (1964): Excavaciones en la villa romana del "Cercado de San Isidro", 
parcela “Villa Possidica”, Dueñas (Palencia). Excavaciones Arqueológicas en España 33. Madrid.

Sanz Mínguez, C.; Campano Lorenzo, A. y Rodríguez Marcos, J.A. (1990): "Nuevos datos sobre la dispersión de la variscita en la Meseta Norte: Las explotaciones de época romana", Actas del Primer Congreso de Historia de Zamora, Tomo 2, Prehistoria-Historia Antigua: 747-764. Zamora.

VV.AA., (1999): Lancia. Historia de la investigación arqueológica. Homenaje a Francisco Jordá Cerdá. León, Diputación Provincial de León.
Villalba, M. J.; Bañolas, L.; Arenas, J. y Alonso, M. (1986): Les mines neolítiques de Can Tintorer, Gavà. Excavacions 1978-1980. Barcelona, Departament de Cultura de la Generalitat de $\mathrm{Ca}-$ talunya.

Villalobos García, R. (2012): “Adornos exóticos en los sepulcros tardoneolíticos de la Submeseta Norte Española. El ejemplo de Las Tuerces como nodo de una red descentralizada de intercambios", en $A c$ tes Xarxes al Neolític: 265-271. Gavà (Barcelona), Museu de Gavà. 